\title{
SINGULAR VARIATION OF DOMAIN AND SPECTRA OF THE LAPLACIAN WITH SMALL ROBIN CONDITIONAL BOUNDARY II
}

Dedicated to Professor Takeshi Watanabe on his 60th birthday

\author{
By Shin Ozawa and Susumu Roppongi
}

\section{Introduction.}

This paper is a continuation of previous paper [6].

Let $\Omega$ be a bounded domain in $\boldsymbol{R}^{2}$ with smooth boundary $\partial \Omega$. Let $\tilde{w}$ be a fixed point point in $\Omega$. Let $B(\varepsilon, \tilde{w})$ be the disk of radius $\varepsilon$ with the center $\tilde{w}$. We put $\Omega_{\varepsilon}=\Omega \backslash \overline{B(\varepsilon, \tilde{w})}$. Consider the following eigenvalue problem

$$
\begin{gathered}
-\Delta u(x)=\lambda u(x) \quad x \in \Omega_{\varepsilon} \\
u(x)=0 \quad x \in \partial \Omega \\
u(x)+k \varepsilon^{\sigma} \frac{\partial u}{\partial \nu_{x}}(x)=0 \quad x \in \partial B(\varepsilon, \tilde{w}) .
\end{gathered}
$$

Here $k$ denotes the positive constant. And $\sigma$ is a real number. Here $\partial / \partial \nu_{x}$ denotes the derivative along the exterior normal direction with respect to $\Omega_{\varepsilon}$.

Let $\mu_{j}(\varepsilon)>0$ be the $j$-th eigenvalue of (1.1). Let $\mu_{j}$ be the $\jmath$-th eigenvalue of the problem

$$
\begin{gathered}
-\Delta u(x)=\lambda u(x) \quad x \in \Omega \\
u(x)=0 \quad x \in \partial \Omega .
\end{gathered}
$$

Let $G(x, y)$ be the Green function of the Laplacian in $\Omega$ associated with the boundary condition (1.2).

Main aim of this paper is to show the following Theorems. Let $\varphi_{j}(x)$ be the $L^{2}$-normalized eigenfunction associated with $\mu_{\jmath}$. We have the following.

THEOREM 1. Assume that $\mu_{\jmath}$ is a simple eigenvalue. Then,

$$
\mu_{j}(\varepsilon)=\mu_{j}-2 \pi \varphi_{j}(\tilde{w})^{2} /(\log \varepsilon)+O\left(|\log \varepsilon|^{-2}\right),
$$

for $\sigma \geqq 1$.

Received March 24, 1992. 
Theorem 2. Assume that $\mu_{\mathrm{j}}$ is a simple eigenvalue. Then,

where

$$
\begin{aligned}
& \mu_{j}(\varepsilon)=\mu_{j}+Q_{j} \varepsilon^{1-\sigma}+R_{j} \varepsilon^{2}+O\left(\varepsilon^{2-\sigma}\right) \quad(-1<\sigma<0) \\
& \mu_{j}(\varepsilon)=\mu_{j}+R_{j} \varepsilon^{2}+Q_{j} \varepsilon^{1-\sigma}+O\left(\varepsilon^{3}|\log \varepsilon|\right) \quad(-2<\sigma \leqq-1) \\
& \mu_{j}(\varepsilon)=\mu_{j}+R_{j} \varepsilon^{2}+O\left(\varepsilon^{3}|\log \varepsilon|\right) \quad(\sigma \leqq-2),
\end{aligned}
$$

$$
\begin{gathered}
Q_{\jmath}=(2 \pi / k) \varphi_{\jmath}(\tilde{w})^{2} \\
R_{\jmath}=-\pi\left(2\left|\operatorname{grad} \varphi_{j}(\tilde{w})\right|^{2}-\mu_{j} \varphi_{j}(\tilde{w})^{2}\right) .
\end{gathered}
$$

Remark. The case $\sigma \in[0,1)$ is treated in [6]. It is curious to the authors that the asymptotic behaviour of $\mu_{j}(\varepsilon)-\mu_{j}$ is the same when $\sigma \leqq-2$. For the related papers we have Ozawa [7], [8], [9], Rauch-Taylor [10], Besson [3], Chavel [4] and the references in the above papers.

For other related problems on singular variation of domains the readers may be referred to Anné [1], Arrieta, Hale and Han [2], Jimbo [5].

\section{Outline of proof of Theorem 1 and Theorem 2 .}

We introduce the following kernel $p_{\varepsilon}(x, y)$.

$$
\begin{aligned}
p_{\varepsilon}(x, y)=G(x, y) & +g(\varepsilon) G(x, \tilde{w}) G(\tilde{w}, y) \\
& +h(\varepsilon)\left\langle\nabla_{w} G(x, \tilde{w}), \nabla_{w} G(\tilde{w}, y)\right\rangle \\
& +i(\varepsilon)\left\langle H_{w} G(x, \tilde{w}), H_{w} G(\tilde{w}, y)\right\rangle,
\end{aligned}
$$

where

$$
\begin{gathered}
\left\langle\nabla_{w} u(\tilde{w}), \nabla_{w} v(\tilde{w})\right\rangle=\sum_{n=1}^{2} \frac{\partial u}{\partial w_{n}} \frac{\partial v}{\partial w_{n}} \mid w=\tilde{w} \\
\left\langle H_{w} u(\tilde{w}), H_{w} v(\tilde{w})\right\rangle=\sum_{m, n=1}^{2} \frac{\partial^{2} u}{\partial w_{m} \partial w_{n}} \frac{\partial^{2} v}{\partial w_{m} \partial w_{n}} \mid w=\tilde{w}
\end{gathered}
$$

when $w=\left(w_{1}, w_{2}\right)$ is an orthonormal frame of $\boldsymbol{R}^{2}$. Here $g(\boldsymbol{\varepsilon}), h(\boldsymbol{\varepsilon}), i(\boldsymbol{\varepsilon})$ are determined so that

$$
p_{\varepsilon}(x, y)+k \varepsilon^{\sigma} \frac{\partial}{\partial \nu_{x}} p_{\varepsilon}(x, y) \quad x \in \partial B(\varepsilon, \tilde{w})
$$

is small in some sense.

If we put

$$
g(\varepsilon)=-\left(\gamma-(2 \pi)^{-1} \log \varepsilon+k(2 \pi)^{-1} \varepsilon^{\sigma-1}\right)^{-1}
$$




$$
\begin{aligned}
h(\varepsilon) & =\left(k \varepsilon^{\sigma}-\varepsilon\right) /\left((2 \pi \varepsilon)^{-1}+k(2 \pi)^{-1} \varepsilon^{\sigma-2}\right) & & (\sigma<0) \\
& =0 & & (\sigma \geqq 1)
\end{aligned}
$$

and

$$
\begin{aligned}
i(\varepsilon) & =k \varepsilon^{\sigma+1} /\left(\pi^{-1} \varepsilon^{-2}+2 k \pi^{-1} \varepsilon^{\sigma-3}\right) & & (\sigma<0) \\
& =0 & & (\sigma \geqq 1),
\end{aligned}
$$

the above aim for (2.2) to be small is attained. Here

$$
\gamma=\lim _{x \rightarrow \tilde{w}}\left(G(x, \tilde{w})+(2 \pi)^{-1} \log |x-\tilde{w}|\right) .
$$

Let $G_{\varepsilon}(x, y)$ be the Green function of the Laplacian in $\Omega_{\varepsilon}$ associated with the boundary condition (1.1).

We put

$$
\begin{aligned}
(\boldsymbol{G} f)(x) & =\int_{\Omega} G(\boldsymbol{x}, y) f(y) d y \\
\left(\boldsymbol{G}_{\varepsilon} f\right)(x) & =\int_{\Omega_{\varepsilon}} G_{\varepsilon}(x, y) f(y) d y
\end{aligned}
$$

and

$$
\begin{aligned}
\left(\boldsymbol{P}_{\varepsilon} f\right)(x) & =\int_{\Omega_{\varepsilon}} p_{\varepsilon}(x, y) f(y) d y & & (\boldsymbol{\sigma}<0) \\
& =\int_{\Omega} p_{\varepsilon}(x, y) f(y) d y & & (\boldsymbol{\sigma} \geqq 1) .
\end{aligned}
$$

In case of $\boldsymbol{\sigma}<0, \boldsymbol{P}_{\varepsilon}$ cannot operate on $L^{p}(\boldsymbol{\Omega})$ because of the existence of $h(\varepsilon)$-term and $i(\varepsilon)$-term in $(2.1)$.

Let $T$ and $T_{\varepsilon}$ be operators on $\Omega$ and $\Omega_{\varepsilon}$, respectively. Then, $\|T\|_{p},\left\|T_{\varepsilon}\right\|_{p, \varepsilon}$ denote the operator norm on $L^{p}(\Omega), L^{p}\left(\Omega_{\varepsilon}\right)$, respectively. Let $f$ and $f_{\varepsilon}$ be functions on $\Omega$ and $\Omega_{\varepsilon}$, respectively. Then, $\|f\|_{p},\left\|f_{\varepsilon}\right\|_{p, \varepsilon}$ denotes the norm on $L^{p}(\Omega), L^{p}\left(\Omega_{\varepsilon}\right)$, respectively.

At first we outline the proof of Theorem 1. A crucial part of our proof of Theorem 1 is the following.

Theorem 3. Fix $\sigma \geqq 1$. Then, there exısts a constant $C$ such that

$$
\left\|\chi_{\varepsilon} \boldsymbol{P}_{\varepsilon} \chi_{\varepsilon}-\boldsymbol{G}_{s}\right\|_{2, \varepsilon} \leqq C \varepsilon|\log \varepsilon|^{-1}
$$

holds. Here $\chi_{\varepsilon}$ is the characteristic function of $\bar{\Omega}_{\varepsilon}$.

Since $\boldsymbol{G}_{\varepsilon}$ is approximated by $\chi_{\varepsilon} \boldsymbol{P}_{\varepsilon} \chi_{\varepsilon}$ and the difference between $\boldsymbol{P}_{\varepsilon}$ and $\chi_{\varepsilon} \boldsymbol{P}_{\varepsilon} \chi_{\varepsilon}$ is small in some sense, we know that everything reduces to our investigation of the perturbative analysis of $\boldsymbol{G} \rightarrow \boldsymbol{P}_{\varepsilon}$. This is the outline of our proof 
of Theorem 1.

Next we outline the proof of Theorem 2. One important part of our proof of Theorem 2 is the following.

THEOREM 4. Fix $\sigma<0$. Then, there exists a constant $C$ such that

$$
\begin{aligned}
\left\|\left(\boldsymbol{P}_{\varepsilon}-\boldsymbol{G}_{\varepsilon}\right)\left(\chi_{\varepsilon} \varphi_{j}\right)\right\|_{2, \varepsilon} & \leqq C \varepsilon^{2-\sigma} \quad(-1<\boldsymbol{\sigma}<0) \\
& \leqq C \varepsilon^{3}|\log \varepsilon| \quad(\sigma \leqq-1)
\end{aligned}
$$

holds.

We fix $j$ and put

$$
\begin{aligned}
\bar{p}_{\varepsilon}(x, y)=G(x, y) & -\pi \mu_{j} \varepsilon^{2} \cdot G(x, \tilde{w}) G(\tilde{w}, y) \\
& +g(\varepsilon) G(x, \tilde{w}) G(\tilde{u}, y) \\
& +h(\varepsilon)\left\langle\nabla_{w} G(x, \tilde{w}), \nabla_{w} G(\tilde{w}, y)\right\rangle \xi_{\varepsilon}(x) \xi_{\varepsilon}(y) \\
& +i(\varepsilon)\left\langle H_{w} G(x, \tilde{w}), H_{w} G(\tilde{w}, y)\right\rangle \xi_{\varepsilon}(x) \xi_{\varepsilon}(y)
\end{aligned}
$$

where $\xi_{\varepsilon}(x) \in C^{\infty}\left(\boldsymbol{R}^{2}\right)$ satısfies $\left|\xi_{\varepsilon}(x)\right| \leqq 1, \xi_{\varepsilon}(x)=1$ for $x \in \boldsymbol{R}^{2} \backslash \overline{B(\varepsilon, \tilde{w})}, \boldsymbol{\xi}_{\varepsilon}(x)=0$ for $x \in B(\varepsilon / 2, \hat{w})$ and $\xi_{\varepsilon}(x-\tilde{w})$ is rotationary invariant. Furthermore we put

$$
\left(\tilde{\boldsymbol{P}}_{\varepsilon} f\right)(x)=\int_{\Omega} \bar{p}_{\varepsilon}(x, y) f(y) d y .
$$

The other important part of our proof of Theorem 2 is the following.

THEOREM 5. Fix $\sigma<0$. Then, there exist a constant $C$ such that

$$
\begin{aligned}
\left\|\left(\chi_{\varepsilon} \overline{\boldsymbol{P}_{\varepsilon}}-\boldsymbol{P}_{\varepsilon} \chi_{\varepsilon}\right) \varphi_{j}\right\|_{2, \varepsilon} & \leqq C \varepsilon^{2-\sigma} \quad(-1<\sigma<0) \\
& \leqq C \varepsilon^{3}|\log \varepsilon| \quad(\sigma \leqq-1)
\end{aligned}
$$

holds.

Since (2.7) and (2.9) are both $o\left(\varepsilon^{2}\right)$, we know that everything reduces to our investigation of the perturbative analysis of $\boldsymbol{G} \rightarrow \boldsymbol{P}_{\varepsilon}$. This is the outline of our proof of Theorem 2 .

\section{Preliminary Lemmas.}

We write $B(\varepsilon, \tilde{w})=B_{\varepsilon}$. Next Lemma is proved in Ozawa [6].

LEMMA 3.1. Fix $\sigma<1$. Assume that $u_{\varepsilon}(x) \in C^{\infty}\left(\bar{\Omega}_{\varepsilon}\right)$ satisfies

$$
\Delta u_{\varepsilon}(x)=0 \quad x \in \Omega_{\varepsilon}
$$




$$
\begin{gathered}
u_{\varepsilon}(x)=0 \quad x \in \partial \Omega \\
\operatorname{Max}\left\{\left|u_{\varepsilon}(x)+k \varepsilon^{\sigma} \frac{\partial u_{\varepsilon}}{\partial \nu_{x}}(x)\right| ; x \in \partial B_{\varepsilon}\right\}=M_{\varepsilon},
\end{gathered}
$$

then

$$
\left\|u_{\varepsilon}\right\|_{p, \varepsilon} \leqq C \varepsilon^{1-\sigma} M_{\varepsilon} \quad(1 \leqq p<+\infty)
$$

holds for a constant $C$ independent of $\varepsilon$.

Remark. In Ozawa [6], $\sigma \geqq 0$ is assumed. But this assumption is not required to get the above Lemma.

Now we want to estimate $\left\|u_{\varepsilon}\right\|_{p, \varepsilon}$ for $\sigma \geqq 1$ under the same assumption of $u_{\varepsilon}$ as above. We have the following.

Lemma 3.2. Fix $M \in C^{\infty}\left(\partial B_{\varepsilon}\right), \sigma \geqq 1$ and $q>\sigma$. Then there exists at least one solution of

$$
\begin{gathered}
\Delta v_{\varepsilon}(x)=0 \quad x \in \boldsymbol{R}^{2} \backslash \bar{B}_{\varepsilon} \\
v_{\varepsilon}(x)+k \varepsilon^{\sigma} \frac{\partial v_{\varepsilon}}{\partial \nu_{x}}(x)=M(\theta) \quad x=\tilde{w}+\varepsilon(\cos \theta, \sin \theta)
\end{gathered}
$$

satisfying

$$
\begin{gathered}
\left|v_{\varepsilon}(x)\right| \leqq C \varepsilon^{1-\sigma} \operatorname{Max}_{\theta}|M(\theta)|(1+|\log r|) \quad \text { for } r \geqq \varepsilon \\
\left|v_{\varepsilon}(x)\right| \leqq C \operatorname{Max}_{\theta}|M(\theta)|\left(|\log r| /|\log \varepsilon|+\varepsilon^{(1 / 2)(1-\sigma / q)}(r-\varepsilon)^{-1 / 2 q^{\prime}}\right)
\end{gathered}
$$

for $r>\varepsilon$, where $r=|x-\tilde{w}|$ and $q^{\prime}$ satisfies $(1 / q)+\left(1 / q^{\prime}\right)=1$.

Proof. We put $x=\tilde{w}+r(\cos \theta, \sin \theta)$ and

$$
v_{\varepsilon}(x)=a_{0} \log r+\sum_{j=1}^{\infty}\left(b_{\jmath} \sin \jmath \theta+c_{\jmath} \cos j \theta\right)(-j)^{-1} r^{-\jmath} .
$$

Then it satisfies $\Delta v_{\varepsilon}(x)=0$ for $x \in \boldsymbol{R}^{2} \backslash \bar{B}_{\varepsilon}$. We see that

$$
v_{\varepsilon}(x)+\left.k \varepsilon^{\sigma} \frac{\partial v_{\varepsilon}}{\partial \nu_{x}}(x)\right|_{x \in \partial B_{\varepsilon}}=s_{0}+\sum_{j=1}^{\infty}\left(s_{\jmath} \sin j \theta+t, \cos j \theta\right)=M(\theta)
$$

implies

for $j \geqq 1$.

$$
\begin{aligned}
& a_{0}\left(\log \varepsilon-k \varepsilon^{\sigma-1}\right)=s_{0} \\
& b_{j} \varepsilon^{-\jmath}\left(-(1 / j)-k \varepsilon^{\sigma-1}\right)=s_{\jmath} \\
& c_{j} \varepsilon^{-\jmath}\left(-(1 / j)-k \varepsilon^{\sigma-1}\right)=t_{\jmath}
\end{aligned}
$$

Thus we have 
408 SHIN OZAWA AND SUSUMU ROPPONGI

$$
\begin{aligned}
\left|v_{\varepsilon}(x)\right| \leqq & \left|s_{0} \log r\right| /\left(k \varepsilon^{\sigma-1}+|\log \varepsilon|\right) \\
& +\left(\sum_{\jmath=1}^{\infty}\left(s_{\jmath}{ }^{2}+t_{\jmath}{ }^{2}\right)\right)^{1 / 2}\left(\sum_{\jmath=1}^{\infty}(\varepsilon / r)^{2 \jmath}\left(1+j k \varepsilon^{\sigma-1}\right)^{-2}\right)^{1 / 2} .
\end{aligned}
$$

Using the Hölder's inequality, we have

$$
\begin{aligned}
& \sum_{j=1}^{\infty}(\varepsilon / r)^{2 \jmath}\left(1+j k \varepsilon^{\sigma-1}\right)^{-2} \\
\leqq & \left(\sum_{\jmath=1}^{\infty}(\varepsilon / r)^{2 \jmath q^{\prime}}\right)^{1 / q^{\prime}}\left(\sum_{j=1}^{\infty}\left(1+\jmath k \varepsilon^{\sigma-1}\right)^{-2 q}\right)^{1 / q} \\
\leqq & \left(\varepsilon^{2 q^{\prime}} /\left(r^{2 q^{\prime}}-\varepsilon^{2 q^{\prime}}\right)\right)^{1 / q^{\prime}}\left(\int_{0}^{\infty}\left(1+k \varepsilon^{\sigma-1} s\right)^{-2 q} d s\right)^{1 / q} \\
\leqq & C(\varepsilon /(r-\varepsilon))^{1 / q^{\prime}} \varepsilon^{-(\sigma-1) / q} \\
= & C \varepsilon^{1-\sigma / q}(r-\varepsilon)^{-1 / q^{\prime}} \quad \text { for } r>\varepsilon .
\end{aligned}
$$

By (3.6), (3.7) and the inequaiity

$$
s_{0}^{2}+\sum_{j=1}^{\infty}\left(s_{j}^{2}+t_{j}^{2}\right) \leqq C \int_{0}^{2 \pi}|M(\theta)|^{2} d \theta \leqq C^{\prime}(\operatorname{Max}|M(\theta)|)^{2},
$$

we get

$$
\begin{aligned}
\left|v_{\varepsilon}(x)\right| \leqq & \left|s_{0}\right| \cdot|\log r| /\left(k \varepsilon^{\sigma-1}\right) \\
& +\left(\sum_{\jmath=1}^{\infty}\left(s_{\jmath}{ }^{2}+t_{\jmath}{ }^{2}\right)\right)^{1 / 2}\left(\sum_{\jmath=1}^{\infty} j^{-2}\right)^{1 / 2} k^{-1} \varepsilon^{1-\sigma} \\
\leqq & C \operatorname{Max}|M(\theta)| \varepsilon^{1-\sigma}(1+|\log r|) \quad \text { for } r \geqq \varepsilon,
\end{aligned}
$$

and

$$
\left|v_{\varepsilon}(x)\right| \leqq C \operatorname{Max}_{\theta}|M(\theta)|\left((|\log r| /|\log \varepsilon|)+\varepsilon^{(1 / 2)(1-\sigma / q)}(r-\varepsilon)^{-1 / 2 q^{\prime}}\right)
$$

for $r>\varepsilon$. Thus the proof is now complete.

q.e.d.

We have the following.

LEMMA 3.3. Fix $\sigma \geqq 1$ and $q>\sigma$. Under the same assumptions of $u_{\varepsilon}$ in Lemma 3.1,

$$
\left\|u_{\varepsilon}\right\|_{p, \varepsilon} \leqq C M_{\varepsilon}\left(|\log \varepsilon|^{-1}+\varepsilon^{(1 / 2)(1-\sigma / q)}\right) \quad\left(1<p<2 q^{\prime}\right)
$$

holds for a constant $C$ independent of $\varepsilon$.

Proof. By Lemma 3.2 and using the same repeating construction of the functions $v_{\varepsilon}^{(n)}$ in Proposition 1 of Ozawa [7], we have

$$
\left|u_{\varepsilon}(x)\right| \leqq C M_{\varepsilon}\left(|\log r| /|\log \varepsilon|+\varepsilon^{(1 / 2)(1-\sigma / q)}(r-\varepsilon)^{-1 / 2 q^{\prime}}\right)
$$


for $r>\varepsilon$.

We fix $R>0$ such that $\Omega \subset B(R, \tilde{w})$. Then, we have

$$
\begin{aligned}
\int_{\Omega_{\varepsilon}}(r-\varepsilon)^{-p / 2 q^{\prime}} d x & \leqq 2 \pi \int_{\varepsilon}^{R} r(r-\varepsilon)^{-p / 2 q^{\prime}} d r \\
& \leqq 2 \pi R \int_{\varepsilon}^{R+\varepsilon}(r-\varepsilon)^{-p / 2 q^{\prime}} d r \leqq C \quad \text { for } 1<p<2 q^{\prime} .
\end{aligned}
$$

By (3.11) and (3.12), we get (3.10).

q.e.d.

\section{Proof of Theorem 3.}

From this section to section 7 , we assume $\sigma \geqq 1$. By (2.3) we know that

$$
g(\varepsilon)=2 \pi(\log \varepsilon)^{-1}+O\left(|\log \varepsilon|^{-2}\right) .
$$

We take an arbitrary fixed point $x \in \partial B_{\varepsilon}$. Without loss of generality we may assume that $\tilde{w}=(0,0)$ and $x=(\varepsilon, 0)$.

We put

$$
S(x, y)=G(x, y)+(1 / 2 \pi) \log |x-y| .
$$

Then, $S(x, y) \in C^{\infty}(\Omega \times \Omega)$.

We put $p_{\varepsilon}(x, y)$ as before. Then, we have

$$
\begin{aligned}
& p_{\varepsilon}(x, y)-\left.k \varepsilon^{\sigma} \frac{\partial}{\partial x_{1}} p_{\varepsilon}(x, y)\right|_{x=(\varepsilon, 0)} \\
& =G(x, y)-k \varepsilon^{\sigma} \frac{\partial}{\partial x_{1}} G(x, y)-g(\varepsilon) k \varepsilon^{\sigma} \frac{\partial}{\partial x_{1}} S(x, \tilde{w}) G(\tilde{w}, y) \\
& \quad+g(\varepsilon)\left(-(2 \pi)^{-1} \log \varepsilon+S(x, \tilde{w})+k(2 \pi)^{-1} \varepsilon^{\sigma-1}\right) G(\tilde{w}, y) .
\end{aligned}
$$

Let $\gamma=S(\tilde{w}, \tilde{w})$. Then, $S(x, \tilde{w})=\gamma+O(\varepsilon)$ as $\varepsilon \rightarrow 0$. Since

$$
g(\varepsilon)\left(-(2 \pi)^{-1} \log \varepsilon+\gamma+k(2 \pi)^{-1} \varepsilon^{\sigma-1}\right)=-1,
$$

we get the following.

$$
\begin{aligned}
p_{\varepsilon}(x, y)-\left.k \varepsilon^{\sigma} \frac{\partial}{\partial x_{1}} p_{\varepsilon}(x, y)\right|_{x=(\varepsilon, 0)} \\
=G(x, y)-G(\tilde{w}, y)-k \varepsilon^{\sigma} \frac{\partial}{\partial x_{1}} G(x, y) \\
\quad+g(\varepsilon)\left(O(\varepsilon)-k \varepsilon^{\sigma} \frac{\partial}{\partial x_{1}} S(x, \tilde{w})\right) G(\tilde{w}, y) .
\end{aligned}
$$

We take an arbitrary $f \in L^{p}\left(\Omega_{\varepsilon}\right)$ and put $\tilde{f}=\chi_{\varepsilon} f$. From (4.2), we get 


$$
\begin{aligned}
\left(\boldsymbol{P}_{\varepsilon} \tilde{f}\right)(x)-\left.k \varepsilon^{\sigma} \frac{\partial}{\partial x_{1}}\left(\boldsymbol{P}_{\varepsilon} \tilde{f}\right)(x)\right|_{x=(\varepsilon, 0)} \\
=(\boldsymbol{G} \tilde{f})(x)-(\boldsymbol{G} \tilde{f})(\tilde{w})-k \boldsymbol{\varepsilon}^{\sigma} \frac{\partial}{\partial x_{1}}(\boldsymbol{G} \tilde{f})(x) \\
\quad+g(\boldsymbol{\varepsilon})\left(O(\boldsymbol{\varepsilon})-k \boldsymbol{\varepsilon}^{\sigma} \frac{\partial}{\partial x_{1}} S(x, w)\right)(\boldsymbol{G} \tilde{f})(\tilde{w}) .
\end{aligned}
$$

By the Sobolev embedding theorem

$$
\|\boldsymbol{G} \tilde{f}\|_{C^{1+\tau(\Omega)}} \leqq C\|\tilde{f}\|_{p}=C\|f\|_{p, \varepsilon}
$$

if $\tau=1-2 / p, 2<p<\infty$. Therefore we get

$$
\begin{aligned}
& |(\boldsymbol{G} \tilde{f})(x)-(\boldsymbol{G} \tilde{f})(\tilde{w})| \leqq C \boldsymbol{\varepsilon}\|f\|_{p, \varepsilon} \\
& |(\boldsymbol{G} \tilde{f})(\tilde{w})| \leqq C\|f\|_{p, \varepsilon} \\
& \left|\frac{\partial}{\partial x_{1}}(\boldsymbol{G} \tilde{f})(x)\right| \leqq C\|f\|_{p, \varepsilon}
\end{aligned}
$$

for $p>2, x=(\varepsilon, 0)$ and $\tilde{w}=(0,0)$.

From (4.1), (4.3) and (4.4) we have the following.

$$
\begin{aligned}
& \left|\left(\boldsymbol{P}_{\varepsilon} \tilde{f}\right)(x)-k \varepsilon^{\sigma} \frac{\partial}{\partial x_{1}}\left(\boldsymbol{P}_{\varepsilon} \tilde{f}\right)(x)\right|_{x=(\varepsilon, 0)} \mid \\
& \quad \leqq C\left(\varepsilon+\varepsilon^{\sigma}+|g(\varepsilon)|\left(\varepsilon+\varepsilon^{\sigma}\right)\right)\|f\|_{p, \varepsilon} \\
& \quad \leqq C \varepsilon\|f\|_{p, \varepsilon} .
\end{aligned}
$$

We put $\left(\boldsymbol{\chi}_{\varepsilon} \boldsymbol{P}_{\varepsilon} \boldsymbol{\chi}_{\varepsilon}-\boldsymbol{G}_{\varepsilon}\right) f=v$. Then, $v=\chi_{\varepsilon} \boldsymbol{P}_{\varepsilon} \tilde{f}-\boldsymbol{G}_{\varepsilon} f$ and $v$ satisfies the assumptions in Lemma 3.3 with $M_{\varepsilon}=C \varepsilon\|f\|_{p, \varepsilon}$, because $G_{\varepsilon} f$ satisfies the given Robin condition on $\partial B_{\varepsilon}$. By Lemma 3.3 we have

$$
\begin{aligned}
\|v\|_{p, \varepsilon} & \leqq C\left(|\log \varepsilon|^{-1}+\varepsilon^{(1 / 2)(1-\sigma / q)}\right) \varepsilon\|f\|_{p, \varepsilon} \\
& \leqq C \varepsilon|\log \varepsilon|^{-1}\|f\|_{p, \varepsilon}
\end{aligned}
$$

for $p>2$ and $q>\sigma$. Therefore,

$$
\left\|\chi_{\varepsilon} \boldsymbol{P}_{\varepsilon} \chi_{\varepsilon}-\boldsymbol{G}_{\varepsilon}\right\|_{p, \varepsilon} \leqq C \varepsilon|\log \varepsilon|^{-1}
$$

for $p>2$.

By the duality argument

$$
\left\|\chi_{\varepsilon} \boldsymbol{P}_{\varepsilon} \chi_{\varepsilon}-\boldsymbol{G}_{\varepsilon}\right\|_{p^{\prime}, \varepsilon} \leqq C \varepsilon|\log \varepsilon|^{-1}
$$

for $p^{\prime}$ satisfying $(1 / p)+\left(1 / p^{\prime}\right)=1$. Now by the Riesz-Thorin interpolation theorem we get Theorem 3 . 


\section{Convergence of eigenvalues for $\sigma \geqq 1$.}

At first we want to estimate $\left\|\boldsymbol{P}_{s}-\boldsymbol{G}\right\|_{2}$. We take an arbitrary $v \in L^{2}(\boldsymbol{\Omega})$. Then, by the definition and the Sobolev embedding theorem we have

$$
\left(\boldsymbol{P}_{\varepsilon} v\right)(x)=(\boldsymbol{G} v)(x)+g(\boldsymbol{\varepsilon}) G(x, \tilde{w})(\boldsymbol{G} v)(\tilde{w})
$$

$$
\|\boldsymbol{G} v\|_{\infty} \leqq C\|v\|_{2} \text {. }
$$

Thus,

$$
\begin{aligned}
\left\|\left(\boldsymbol{P}_{\varepsilon}-\boldsymbol{G}\right) v\right\|_{2} & \leqq C|g(\boldsymbol{\varepsilon})|\|G(\cdot, \tilde{w})\|_{2}\|v\|_{2} \\
& \leqq C|g(\varepsilon)|\|v\|_{2} \leqq C|\log \varepsilon|^{-1}\|v\|_{2} .
\end{aligned}
$$

Therefore we get the following.

LEMMA 5.1. There exists a constants $C$ independent of $\varepsilon$ such that

$$
\left\|\boldsymbol{P}_{\varepsilon}-\boldsymbol{G}\right\|_{2} \leqq C|\log \varepsilon|^{-1}
$$

holds.

Next we want to estimate $\left\|\boldsymbol{P}_{\varepsilon}-\chi_{\varepsilon} \boldsymbol{P}_{\varepsilon} \chi_{\varepsilon}\right\|_{2}$. Since

$$
\boldsymbol{P}_{\varepsilon}-\chi_{\varepsilon} \boldsymbol{P}_{\varepsilon} \chi_{\varepsilon}=\left(1-\chi_{\varepsilon}\right) \boldsymbol{P}_{\varepsilon} \chi_{\varepsilon}+\boldsymbol{P}_{\varepsilon}\left(1-\chi_{\varepsilon}\right),
$$

we have

$$
\left\|\boldsymbol{P}_{\varepsilon}-\chi_{\varepsilon} \boldsymbol{P}_{\varepsilon} \chi_{\varepsilon}\right\|_{2} \leqq\left\|\left(1-\chi_{\varepsilon}\right) \boldsymbol{P}_{\varepsilon} \chi_{\varepsilon}\right\|_{2}+\left\|\boldsymbol{P}_{\varepsilon}\left(1-\chi_{\varepsilon}\right)\right\|_{2} .
$$

By (5.1) and (5.2) we have

$$
\begin{aligned}
\left\|\left(1-\chi_{\varepsilon}\right)\left(\boldsymbol{P}_{\varepsilon} v\right)\right\|_{2} & \leqq\left\|\left(1-\chi_{\varepsilon}\right)(\boldsymbol{G} v)\right\|_{2}+|g(\varepsilon)|\left\|\left(1-\chi_{\varepsilon}\right) G(\cdot, \tilde{w})(\boldsymbol{G} v)(\tilde{w})\right\|_{2} \\
& \leqq C\left|B_{\varepsilon}\right|^{1 / 2}\|v\|_{2}+C|g(\varepsilon)|\left(\int_{B_{\varepsilon}}|G(x, \tilde{w})|^{2} d x\right)^{1 / 2}\|v\|_{2} \\
& \leqq C(\varepsilon+|g(\varepsilon)| \varepsilon|\log \varepsilon|)\|v\|_{2} \\
& \leqq C \varepsilon\|v\|_{2} .
\end{aligned}
$$

Therefore we get

$$
\begin{gathered}
\left\|\left(1-\chi_{\varepsilon}\right) \boldsymbol{P}_{\varepsilon}\right\|_{2} \leqq C \varepsilon \\
\left\|\left(1-\chi_{\varepsilon}\right) \boldsymbol{P}_{\varepsilon} \chi_{\varepsilon}\right\|_{2} \leqq C \varepsilon
\end{gathered}
$$

Since we have the duality

$$
\left(\left(1-\chi_{\varepsilon}\right) \boldsymbol{P}_{\varepsilon}\right)^{*}=\boldsymbol{P}_{\varepsilon}\left(1-\chi_{\varepsilon}\right)
$$

we get

$$
\left\|\boldsymbol{P}_{\varepsilon}\left(1-\chi_{\varepsilon}\right)\right\|_{2} \leqq C \varepsilon
$$


By (5.4), (5.5), (5.6) we get the following.

LEMMA 5.2. There exists a constant $C$ independent of $\varepsilon$ such that

$$
\left\|\boldsymbol{P}_{\varepsilon}-\chi_{\varepsilon} \boldsymbol{P}_{\varepsilon} \chi_{\varepsilon}\right\|_{2} \leqq C \varepsilon
$$

holds.

By virtue of Theorem 3, Lemma 5.1, Lemma 5.2, we see that there exists a constant $C$ independent of $j$ such that

$$
\begin{aligned}
\left|\mu_{j}(\varepsilon)^{-1}-\mu_{j}^{-1}\right| & \leqq C\left(\varepsilon|\log \varepsilon|^{-1}+|\log \varepsilon|^{-1}+\varepsilon\right) \\
& \leqq C|\log \varepsilon|^{-1}
\end{aligned}
$$

holds.

We need more precise estimate for the left hand side of (5.7) to get Theorem 1. By (5.7) we know that the multiplicity of $\mu_{j}(\varepsilon)$ is one for small $\varepsilon$ when the multiplicity of $\mu_{\text {o }}$ is one.

\section{Perturbational Calculus for $\boldsymbol{P}_{\varepsilon}$.}

In this section we consider the behaviour of eigenvalues of $\boldsymbol{P}_{\varepsilon}$ as $\boldsymbol{\varepsilon}$ tends to 0 .

We put $A_{0}=G$ and

$$
\left(A_{1} f\right)(x)=G(x, \tilde{w})(\boldsymbol{G} f)(\tilde{w}) .
$$

Then,

It is easy to see

$$
\boldsymbol{P}_{\varepsilon}=A_{0}+g(\varepsilon) A_{1} .
$$

$$
\left\|A_{1}\right\|_{p} \leqq C \quad(1<p<\infty)
$$

Furthermore we put

$$
\begin{aligned}
& \lambda(\varepsilon)=\lambda_{0}+g(\varepsilon) \lambda_{1} \\
& \phi(\varepsilon)=\phi_{0}+g(\varepsilon) \phi_{1}
\end{aligned}
$$

so that $\lambda(\varepsilon)$ and $\phi(\varepsilon)$ is an approximate eigenvalue of $\boldsymbol{P}_{\varepsilon}$ and an approximate eigenfunction of $\boldsymbol{P}_{\varepsilon}$, respectively.

As the standard techniques of perturbation theory, we solve the following equations.

Let $\lambda_{0}$ be a simple eigenvalue of $A_{0}$. At first

$$
\left(A_{0}-\lambda_{0}\right) \psi_{0}=0, \quad\left\|\phi_{0}\right\|_{2}=1 .
$$

Next we solve the following equations;

$$
\left(A_{0}-\lambda_{0}\right) \psi_{1}=\left(\lambda_{1}-A_{1}\right) \psi_{0}
$$




$$
\left(\psi_{0}, \phi_{1}\right)_{2}=0 \text {, }
$$

where $(,)_{2}$ denotes the inner product on $L^{2}(\Omega)$.

By the Fredholm alternative theory, we see that

$$
\lambda_{1}=\left(A_{1} \psi_{0}, \phi_{0}\right)_{2}
$$

is the condition such that the unique solution $\phi_{1}$ of (6.2), (6.3) exists.

Hereafter we put $\lambda_{0}=\mu_{\jmath}^{-1}$. Then $\phi_{0}=\varphi_{\jmath}$. We see that

$$
\lambda_{1}=\left|\left(\boldsymbol{G} \psi_{0}\right)(\tilde{w})\right|^{2}=\mu_{j}^{-2} \varphi_{j}(\tilde{w})^{2}
$$

$$
\left(\boldsymbol{P}_{\varepsilon}-\lambda(\varepsilon)\right) \psi(\varepsilon)=g(\varepsilon)^{2}\left(A_{1}-\lambda_{1}\right) \psi_{1} .
$$

By the Fredholm theory, we see that

$$
\left\|\psi_{1}\right\|_{2} \leqq C\left\|\lambda_{1}-A_{1}\right\|_{2}\left\|\psi_{0}\right\|_{2} \leqq C
$$

By (6.6), (6.7), we have

$$
\begin{aligned}
\left\|\left(\boldsymbol{P}_{\varepsilon}-\lambda(\varepsilon)\right) \psi(\varepsilon)\right\|_{2} & \leqq|g(\boldsymbol{\varepsilon})|^{2}\left\|A_{1}-\lambda_{1}\right\|_{2}\left\|\phi_{1}\right\|_{2} \\
& \leqq C|g(\varepsilon)|^{2} \leqq C|\log \varepsilon|^{-2} .
\end{aligned}
$$

Therefore, we get the following.

LEMMA 6.1. There exists a constant $C$ independent of $\varepsilon$ such that

$$
\left\|\left(\boldsymbol{P}_{\varepsilon}-\lambda(\varepsilon)\right) \psi(\varepsilon)\right\|_{2} \leqq C|\log \varepsilon|^{-2}
$$

holds.

Next we want to estimate $\left\|\left(\boldsymbol{P}_{\varepsilon}-\lambda(\varepsilon)\right)\left(1-\chi_{\varepsilon}\right) \psi(\varepsilon)\right\|_{2, \varepsilon}$. We put $\hat{\chi}_{\varepsilon}=1-\chi_{\varepsilon} . \quad$ Then, we have

$$
\left(\boldsymbol{P}_{\varepsilon}-\lambda(\varepsilon)\right) \hat{\chi}_{\varepsilon} \psi(\varepsilon)=\sum_{h=1}^{4} T_{h},
$$

where

$$
\begin{aligned}
& T_{1}=G \hat{\chi}_{\varepsilon} \psi_{0} \\
& T_{2}=g(\varepsilon) G \hat{\chi}_{\varepsilon} \psi_{1} \\
& T_{3}=g(\varepsilon) A_{1} \hat{\chi}_{\varepsilon} \psi_{0} \\
& T_{4}=g(\varepsilon)^{2} A_{1} \hat{\chi}_{\varepsilon} \psi_{1}
\end{aligned}
$$

on $\Omega_{\varepsilon}$, since $\lambda(\varepsilon) \hat{\chi}_{\varepsilon} \psi(\varepsilon)=0$ on $\Omega_{\varepsilon}$.

We get

$$
\left\|T_{1}\right\|_{2, \varepsilon} \leqq\left\|T_{1}\right\|_{\infty} \leqq C \cdot\left\|\hat{\chi}_{\varepsilon} \varphi_{J}\right\|_{2} \leqq C \varepsilon
$$

Also, 


$$
\left\|T_{2}\right\|_{2, \varepsilon} \leqq C|g(\varepsilon)| \cdot\left\|\hat{\chi}_{\varepsilon} \psi_{1}\right\|_{2} .
$$

Notice that

$$
\phi_{1}=\left(-\lambda_{0}\right)^{-1}\left(\left(\lambda_{1}-A_{1}\right) \phi_{0}-A_{0} \phi_{1}\right)
$$

Then,

$$
\begin{aligned}
\left\|\hat{\chi}_{\varepsilon} \psi_{1}\right\|_{2} & \leqq C\left(\left\|\hat{\chi}_{\varepsilon} \psi_{0}\right\|_{2}+\left\|\hat{\chi}_{\varepsilon} A_{1} \psi_{0}\right\|_{2}+\left\|\hat{\chi}_{\varepsilon} A_{0} \psi_{1}\right\|_{2}\right) \\
& \leqq C\left(\left\|\hat{\chi}_{\varepsilon}\right\|_{2}+\left(\int_{B_{\varepsilon}}|G(x, \tilde{w})|^{2} d x\right)^{1 / 2}+\left\|\hat{\chi}_{\varepsilon}\right\|_{2}\right) \\
& \leqq G(\varepsilon+\varepsilon|\log \varepsilon|+\varepsilon) \leqq C \varepsilon|\log \varepsilon| .
\end{aligned}
$$

Therefore, we get

$$
\left\|T_{2}\right\|_{2, \varepsilon} \leqq C|g(\varepsilon)| \varepsilon|\log \varepsilon| \leqq C \varepsilon .
$$

Furthermore, we have

$$
\begin{aligned}
\left\|T_{3}+T_{4}\right\|_{2, \varepsilon} & \leqq \\
& \leqq\left. C(\varepsilon)\left|\left\|A_{1} \hat{\chi}_{\varepsilon} \psi_{0}\right\|_{2}+\right| g(\varepsilon)\right|^{2}\left\|A_{1} \hat{\chi}_{\varepsilon} \psi_{1}\right\|_{2} \\
& \leqq C\left(\varepsilon|\log \varepsilon|^{-1}+|\log \varepsilon|^{-2} \|_{2}+|g(\varepsilon)|^{2}\right) \\
& \leqq C|\log \varepsilon|^{-2} .
\end{aligned}
$$

Summing up (6.10), (6.11), (6.12) and (6.13), we have the following inequality.

$$
\|(6.10)\|_{2, \varepsilon} \leqq C\left(\varepsilon+\varepsilon+|\log \varepsilon|^{-2}\right) \leqq C|\log \varepsilon|^{-2} .
$$

Therefore, we get the following.

LEMMA 6.2. There exists a constant $C$ independent of $\varepsilon$ such that

$$
\left\|\left(\boldsymbol{P}_{\varepsilon}-\lambda(\varepsilon)\right)\left(1-\chi_{\varepsilon}\right) \psi(\varepsilon)\right\|_{2, \varepsilon} \leqq C|\log \varepsilon|^{-2}
$$

holds.

\section{Proof of Theorem 1.}

Now we are in a position to prove Theorem 1. By Theorem 3, Lemma 6.1 and 6.2 , we have

$$
\begin{aligned}
\left\|\left(\boldsymbol{G}_{\varepsilon}-\lambda(\varepsilon)\right)\left(\chi_{\varepsilon} \psi(\varepsilon)\right)\right\|_{2, \varepsilon} \leqq & \left\|\boldsymbol{G}_{\varepsilon}-\chi_{\varepsilon} \boldsymbol{P}_{\varepsilon} \chi_{\varepsilon}\right\|_{2, \varepsilon}\|\phi(\varepsilon)\|_{2, \varepsilon}+\left\|\left(\boldsymbol{P}_{\varepsilon}-\lambda(\varepsilon)\right) \psi(\varepsilon)\right\|_{2, \varepsilon} \\
& +\left\|\left(\boldsymbol{P}_{\varepsilon}-\lambda(\varepsilon)\right)\left(1-\chi_{\varepsilon}\right) \psi(\varepsilon)\right\|_{2, \varepsilon} \\
\leqq & C\left(\varepsilon|\log \varepsilon|^{-1}\|\psi(\varepsilon)\|_{2, \varepsilon}+|\log \varepsilon|^{-2}+|\log \varepsilon|^{-2}\right) \\
\leqq & C|\log \varepsilon|^{-2} .
\end{aligned}
$$


Here we used the fact that $\|\psi(\varepsilon)\|_{2, \varepsilon} \in(1 / 2,2)$ for small $\varepsilon$. Therefore, there exists at least one eigenvalue $\lambda^{*}(\varepsilon)$ of $G_{\varepsilon}$ satisfying

$$
\left|\lambda^{*}(\varepsilon)-\lambda(\varepsilon)\right| \leqq C|\log \varepsilon|^{-2} .
$$

We here represent $\lambda(\varepsilon)$ explicitly as follows :

$$
\begin{aligned}
\lambda(\varepsilon) & =\mu_{\jmath}^{-1}+g(\varepsilon) \mu_{\jmath}^{-2} \varphi_{j}(\tilde{w})^{2} \\
& =\mu_{\jmath}^{-1}+2 \pi \mu_{\jmath}^{-2} \varphi_{\jmath}(\tilde{w})^{2}(\log \varepsilon)^{-1}+O\left(|\log \varepsilon|^{-2}\right) .
\end{aligned}
$$
we get

By (7.1), (7.2) and the fact (5.7), we see that $\lambda^{*}(\varepsilon)$ must be $\mu_{j}(\varepsilon)^{-1}$. Then,

$$
\left|\mu_{j}(\varepsilon)^{-1}-\left(\mu_{j}^{-1}+2 \pi \mu_{j}^{-2} \varphi_{j}(\tilde{w})^{2}(\log \varepsilon)^{-1}\right)\right| \leqq C|\log \varepsilon|^{-2} .
$$

Therefore, we get the desired Theorem 1 .

\section{Proof of Theorem 4.}

From this section we assume $\sigma<0$. By (2.3), (2.4) and (2.5), we see that

$$
\begin{gathered}
g(\varepsilon)=-(2 \pi / k) \varepsilon^{1-\sigma}+O\left(\varepsilon^{2-2 \sigma}|\log \varepsilon|\right) \\
h(\varepsilon)=2 \pi \varepsilon^{2}+O\left(\varepsilon^{3-\sigma}\right) \\
i(\varepsilon)=(\pi / 2) \varepsilon^{4}+O\left(\varepsilon^{5-\sigma}\right) .
\end{gathered}
$$

At first we want to estimate $\left\|\boldsymbol{P}_{\varepsilon}-\boldsymbol{G}_{\varepsilon}\right\|_{2, \varepsilon}$. We take an arbitrary fixed point $x \in \partial B_{\varepsilon}$. Without loss of generality we may assume that $\tilde{w}=(0,0)$ and $x=$ $(\varepsilon, 0)$.

We put $S(x, y)$ as before. Then, we have the following formulas (8.2), (8.3) in p. 263 and (8.4) in p. 264 of Ozawa [7], respectively.

$$
\begin{aligned}
& \left\langle\nabla_{w} G(x, \tilde{w}), \nabla_{w} G(\tilde{w}, y)\right\rangle \\
& \quad=(2 \pi \varepsilon)^{-1} \frac{\partial}{\partial w_{1}} G(\tilde{w}, y)+\left\langle\nabla_{w} S(x, \tilde{w}), \nabla_{w} G(\tilde{w}, y)\right\rangle
\end{aligned}
$$

for $x=(\varepsilon, 0), \tilde{w}=(0,0)$.

$$
\begin{aligned}
\frac{\partial}{\partial x_{1}} & \left\langle\nabla_{w} G(x, \tilde{w}), \nabla_{w} G(\tilde{w}, y)\right\rangle \\
& =-(2 \pi)^{-1} \varepsilon^{-2} \frac{\partial}{\partial w_{1}} G(\tilde{w}, y)+\frac{\partial}{\partial x_{1}}\left\langle\nabla_{w} S(x, \tilde{w}), \nabla_{w} G(\tilde{w}, y)\right\rangle
\end{aligned}
$$

for $x=(\varepsilon, 0), \tilde{w}=(0,0)$. 


$$
\begin{aligned}
& \frac{\partial}{\partial x_{1}}\left\langle H_{w} G(x, \tilde{w}), H_{w} G(\tilde{w}, y)\right\rangle \\
& \quad=-2 \pi^{-1} \varepsilon^{-3} \frac{\partial^{2}}{\partial w_{1}^{2}} G(\tilde{w}, y)+\frac{\partial}{\partial x_{1}}\left\langle H_{w} S(x, \tilde{w}), H_{w} G(\tilde{w}, y)\right\rangle
\end{aligned}
$$

for $x=(\varepsilon, 0), \tilde{u}=(0,0)$.

The same calculation yields

$$
\begin{aligned}
& \left\langle H_{w} G(x, \tilde{w}), H_{w} G(\tilde{w}, y)\right\rangle \\
& \quad=\pi^{-1} \varepsilon^{-2} \frac{\partial^{2}}{\partial w_{1}^{2}} G\left(\tilde{w^{\prime}}, y\right)+\left\langle H_{w} S(x, \tilde{w}), H_{w} G(\tilde{w}, y)\right\rangle
\end{aligned}
$$

for $x=(\varepsilon, 0), \tilde{w}=(0,0)$.

We put $p_{\varepsilon}(x, y)$ as before. By (8.2), (8.3), (8.4) and (8.5), we have

$$
p_{\varepsilon}(x, y)-\left.k \varepsilon^{\sigma} \frac{\partial}{\partial x_{1}} p_{\varepsilon}(x, y)\right|_{x=(\varepsilon, 0)}=\sum_{j=1}^{7} L_{\jmath},
$$

where

$$
\begin{aligned}
L_{1}= & G(x, y) \\
L_{2}= & g(\varepsilon)\left(-(2 \pi)^{-1} \log \varepsilon+\gamma+(2 \pi)^{-1} k \varepsilon^{\sigma-1}\right) G\left(\tilde{u^{\prime}}, y\right) \\
L_{3}= & g(\varepsilon)\left(O(\varepsilon)-k \varepsilon^{\sigma-1} \frac{\partial}{\partial x_{1}} S(x, \tilde{w})\right) G(\tilde{w}, y) \\
L_{4}= & (2 \pi)^{-1}\left(\varepsilon^{-1}+k \varepsilon^{\sigma-1}\right) h(\varepsilon) \frac{\partial}{\partial w_{1}} G(\tilde{w}, y)-k \varepsilon^{\sigma} \frac{\partial}{\partial x_{1}} G(x, y) \\
L_{5}= & \pi^{-1}\left(\varepsilon^{-2}+2 k \varepsilon^{\sigma-3}\right) i(\varepsilon) \frac{\partial^{2}}{\partial w_{1}{ }^{2}} G(\tilde{w}, y) \\
L_{6}= & h(\varepsilon)\left\langle\nabla_{w} S(x, \tilde{w}), \nabla_{w} G(\tilde{w}, y)\right\rangle \\
& -k \varepsilon^{\sigma} h(\varepsilon) \frac{\partial}{\partial x_{1}}\left\langle\nabla_{w} S(x, \tilde{w}), \nabla_{w} G(\tilde{w}, y)\right\rangle \\
L_{7}= & i(\varepsilon)\left\langle H_{w} S(x, \tilde{w}), H_{w} G(\tilde{w}, y)\right\rangle \\
& -k \varepsilon^{\sigma} i(\varepsilon) \frac{\partial}{\partial x_{1}}\left\langle H_{w} S(x, \tilde{w}), H_{w} G(\tilde{w}, y)\right\rangle
\end{aligned}
$$

for $x=(\varepsilon, 0), \tilde{w}=(0,0)$.

Here we used the fact that

$$
S(x, \tilde{w})=\gamma+O(\varepsilon) \quad \text { as } \varepsilon \rightarrow 0 .
$$

By (2.3), (2.4), (2.5) and (8.6), we get the following. 


$$
\begin{aligned}
& p_{\varepsilon}(x, y)-\left.k \varepsilon^{\sigma} \frac{\partial}{\partial x_{1}} p_{\varepsilon}(x, y)\right|_{x=(\varepsilon, 0)} \\
&= G(x, y)-G(\tilde{w}, y)-\varepsilon \frac{\partial}{\partial w_{1}} G(\tilde{w}, y) \\
&+g(\varepsilon)\left(O(\varepsilon)-k \varepsilon^{\sigma} \frac{\partial}{\partial x_{1}} S(x, \tilde{w})\right) G(\tilde{w}, y) \\
&-k \varepsilon^{\sigma}\left(\frac{\partial}{\partial x_{1}} G(x, y)-\frac{\partial}{\partial w_{1}} G(\tilde{w}, y)-\varepsilon \frac{\partial^{2}}{\partial w_{1}^{2}} G(\tilde{w}, y)\right) \\
&+L_{6}+L_{7} .
\end{aligned}
$$

We take an arbitrary $\tilde{f} \in L^{p}(\Omega)$ which is zero on $B_{\varepsilon}$. By (8.7), we have

$$
\begin{aligned}
\boldsymbol{P}_{\varepsilon} \tilde{f}(x)-\left.k \varepsilon^{\sigma} \frac{\partial}{\partial x_{1}}\left(\boldsymbol{P}_{\varepsilon} \tilde{f}\right)(x)\right|_{x=(\varepsilon, 0)} \\
=(\boldsymbol{G} \tilde{f})(x)-(\boldsymbol{G} \tilde{f})(\tilde{w})-\varepsilon \frac{\partial}{\partial w_{1}}(\boldsymbol{G} \tilde{f})(\tilde{w}) \\
\quad+g(\boldsymbol{\varepsilon})\left(O(\varepsilon)-k \varepsilon^{\sigma} \frac{\partial}{\partial x_{1}} S(x, \tilde{w})\right)(\boldsymbol{G} \tilde{f})(\tilde{w}) \\
\quad-k \varepsilon^{\sigma}\left(\frac{\partial}{\partial x_{1}}(\boldsymbol{G} \tilde{f})(x)-\frac{\partial}{\partial w_{1}}(\boldsymbol{G} \tilde{f})(\tilde{w})-\varepsilon \frac{\partial^{2}}{\partial w_{1}{ }^{2}}(\boldsymbol{G} \tilde{f})(\tilde{w})\right) \\
+h(\boldsymbol{\varepsilon})\left\langle\nabla_{w} S(x, \tilde{w}), \nabla_{w}(\boldsymbol{G} \tilde{f})(\tilde{w})\right\rangle \\
\quad-k \varepsilon^{\sigma} h(\varepsilon) \frac{\partial}{\partial x_{1}}\left\langle\nabla_{w} S(x, \tilde{w}), \nabla_{w}(\boldsymbol{G} \tilde{f})(\tilde{w})\right\rangle \\
\quad+i(\boldsymbol{\varepsilon})\left\langle H_{w} S(x, \tilde{w}), H_{w}(\boldsymbol{G} \tilde{f})(\tilde{w})\right\rangle \\
\quad-k \varepsilon^{\sigma} i(\boldsymbol{\varepsilon}) \frac{\partial}{\partial x_{1}}\left\langle H_{w} S(x, \tilde{w}), H_{w}(\boldsymbol{G} \tilde{f})(\tilde{w})\right\rangle .
\end{aligned}
$$

We want to estimate (8.8). By the Sobolev embedding theorem,

$$
\|G \tilde{f}\|_{C^{1+\tau(\Omega)}} \leqq C\|\tilde{f}\|_{p, \varepsilon}
$$

for $p>2, \tau=1-2 / p$.

Therefore, we have

$$
\begin{aligned}
& |(\boldsymbol{G} \tilde{f})(\tilde{w})| \leqq C\|\tilde{f}\|_{p, \varepsilon} \\
& \left|(\boldsymbol{G} \tilde{f})(x)-(\boldsymbol{G} \tilde{f})(\tilde{w})-\varepsilon \frac{\partial}{\partial w_{1}}(\boldsymbol{G} \tilde{f})(\tilde{w})\right| \leqq C \varepsilon^{1+\tau}\|\tilde{f}\|_{p, \varepsilon} \\
& \left|\frac{\partial}{\partial x_{1}}(\boldsymbol{G} \tilde{f})(x)-\frac{\partial}{\partial w_{1}}(\boldsymbol{G} \tilde{f})(\tilde{w})\right| \leqq C \varepsilon^{\tau}\|\tilde{f}\|_{p, \varepsilon}
\end{aligned}
$$


for $p>2, x=(\varepsilon, 0)$.

Furthermore,

$$
\begin{aligned}
\left|\frac{\partial}{\partial w_{n}}(\boldsymbol{G} \tilde{f})(\tilde{w})\right| & \leqq C\left(\int_{\Omega_{\varepsilon}}|y-\tilde{w}|^{-p^{\prime}} d y\right)^{1 / p^{\prime}}\|\tilde{f}\|_{p, \varepsilon} \\
& \leqq C|\log \varepsilon|^{1 / 2}\|\tilde{f}\|_{2, \varepsilon} \quad(p=2) \\
& \leqq C\|\tilde{f}\|_{p, \varepsilon} \quad(p>2)
\end{aligned}
$$

for $n=1,2$, where $p^{\prime}$ satisfies $(1 / p)+\left(1 / p^{\prime}\right)=1$. Also,

$$
\begin{aligned}
\left|\frac{\partial^{2}}{\partial w_{m} \partial w_{n}}(\boldsymbol{G} \tilde{f})(\tilde{w})\right| & \leqq C\left(\int_{\Omega_{\varepsilon}}|y-\tilde{w}|^{-2 p^{\prime}} d y\right)^{1 / p^{\prime}}\|\tilde{f}\|_{p, \varepsilon} \\
& \leqq C \varepsilon^{-2 / p}\|\tilde{f}\|_{p, \varepsilon} \quad(p>1)
\end{aligned}
$$

for $1 \leqq m, n \leqq 2$.

Summing up (8.8), (8.9), (8.10) and (8.11), we get

$$
\begin{aligned}
& \left|\left(\boldsymbol{P}_{\varepsilon} \tilde{f}\right)(x)-k \varepsilon^{\sigma} \frac{\partial}{\partial x_{1}}\left(\boldsymbol{P}_{\varepsilon} \tilde{f}\right)(x)\right|_{x=(\varepsilon, 0)} \mid \\
& \leqq C\left(\varepsilon^{1+\tau}+\varepsilon^{1-\sigma+\sigma}+\varepsilon^{\sigma}\left(\varepsilon^{\tau}+\varepsilon^{1-2 / p}\right)+\varepsilon^{\sigma+2}+\varepsilon^{4+\sigma-2 / p}\right)\|\tilde{f}\|_{p, \varepsilon} \\
& \leqq C \varepsilon^{\sigma+1-2 / p}\|\tilde{f}\|_{p, \varepsilon}
\end{aligned}
$$

for $p>2$.

We put $\left(\boldsymbol{P}_{\varepsilon}-\boldsymbol{G}_{\varepsilon}\right) \tilde{f}=v$. Then, $v$ satisfies the assumption in Lemma 3.1 with $M_{\varepsilon}=C \varepsilon^{\sigma+1-2 / p}\|\tilde{f}\|_{p, \varepsilon}$, because $\boldsymbol{G}_{\varepsilon} \tilde{f}$ satisfies the given Robin condition on $\partial B_{\varepsilon}$. By Lemma 3.1, we have

$$
\|v\|_{p, \varepsilon} \leqq C \varepsilon^{1-\sigma} \varepsilon^{1+\sigma-2 / p}\|\tilde{f}\|_{p, \varepsilon} \leqq C \varepsilon^{2-2 / p}\|\tilde{f}\|_{p, \varepsilon}
$$

for $p>2$. Therefore,

$$
\left\|P_{\varepsilon}-G_{\varepsilon}\right\|_{p, \varepsilon} \leqq C \varepsilon^{2-2 / p} \quad(p>2) .
$$

By the duality argument and the Riesz-Thorin interpolation theorem, we get

$$
\left\|\boldsymbol{P}_{\varepsilon}-\boldsymbol{G}_{\varepsilon}\right\|_{2, \varepsilon} \leqq C \varepsilon^{2-2 / p} \quad(p>2) .
$$

We take an arbitrary $\beta \in(0,1)$ and put $p=2 /(1-\beta)$. Then, we have the following.

PROPOSITION 8.1. Fix $\beta \in(0,1)$. Then, there exists a constant $C$ independent of $\varepsilon$ such that

$$
\left\|P_{\varepsilon}-G_{\varepsilon}\right\|_{2, \varepsilon} \leqq C \varepsilon^{1+\beta}
$$

holds.

Next we estimate $\left\|\left(\boldsymbol{P}_{\varepsilon}-\boldsymbol{G}_{\varepsilon}\right)\left(\chi_{\boldsymbol{\varepsilon}} \varphi_{j}\right)\right\|_{2, \varepsilon}$. We put $\left(\boldsymbol{P}_{\varepsilon}-\boldsymbol{G}_{\varepsilon}\right)\left(\boldsymbol{\chi}_{\varepsilon} \varphi_{j}\right)=v_{\varepsilon}$. As we 
get (8.8), we have

$$
v_{\varepsilon}(x)-\left.k \varepsilon^{\sigma} \frac{\partial v_{\varepsilon}}{\partial x_{1}}(x)\right|_{x=(\varepsilon, 0)}=I_{0}(\varepsilon)-k \varepsilon^{\sigma}\left(I_{1}(\varepsilon)-I_{2}(\varepsilon)\right)+I_{3}(\varepsilon)
$$

where

$$
\begin{aligned}
& I_{0}(\varepsilon)=\left(\boldsymbol{G} \chi_{\varepsilon} \varphi_{j}\right)(x)-\left(\boldsymbol{G} \chi_{\varepsilon} \varphi_{j}\right)(\tilde{w})-\varepsilon_{\partial w_{1}}^{\partial}\left(\boldsymbol{G} \chi_{\varepsilon} \varphi_{j}\right)(\tilde{w}) \\
& I_{1}(\varepsilon)= \frac{\partial}{\partial x_{1}}\left(\boldsymbol{G} \varphi_{j}\right)(x)-\left(\frac{\partial}{\partial w_{1}}+\varepsilon \frac{\partial^{2}}{\partial w_{1}^{2}}\right)\left(\boldsymbol{G} \varphi_{j}\right)(\tilde{w}) \\
& I_{2}(\varepsilon)= \frac{\partial}{\partial x_{1}}\left(\boldsymbol{G} \hat{\chi}_{\varepsilon} \varphi_{j}\right)(x)-\left(\frac{\partial}{\partial w_{1}}+\varepsilon \frac{\partial}{\partial w_{1}^{2}}\right)\left(\boldsymbol{G} \hat{\chi}_{\varepsilon} \varphi_{j}\right)(\tilde{w}) \\
& I_{3}(\varepsilon)= g(\varepsilon)\left(O(\varepsilon)-k \varepsilon^{\sigma} \frac{\partial}{\partial x_{1}} S(x, \tilde{w})\right)\left(\boldsymbol{G} \chi_{\varepsilon} \varphi_{j}\right)(\tilde{w}) \\
&+h(\varepsilon)\left\langle\nabla_{w} S(x, \tilde{w}), \nabla_{w}\left(\boldsymbol{G} \chi_{\varepsilon} \varphi_{j}\right)(\tilde{w})\right\rangle \\
&-k \varepsilon^{\sigma} h(\varepsilon) \frac{\partial}{\partial x_{1}}\left\langle\nabla_{w} S(x, \tilde{u}), \nabla_{w}\left(\boldsymbol{G} \chi_{\varepsilon} \varphi_{j}\right)(\tilde{w})\right\rangle \\
&+i(\varepsilon)\left\langle H_{w} S(x, \tilde{w}), H_{w}\left(\boldsymbol{G} \chi_{\varepsilon} \varphi_{j}\right)(\tilde{w})\right\rangle \\
&-k \varepsilon^{\sigma} i(\varepsilon) \frac{\partial}{\partial x_{1}}\left\langle H_{w} S(x, \tilde{w}), H_{w}\left(\boldsymbol{G} \chi_{\varepsilon} \varphi_{j}\right)(\tilde{w})\right\rangle \\
& \quad \text { for } x=(\varepsilon, 0), \tilde{w}=(0,0) .
\end{aligned}
$$

Here we put $\hat{\chi}_{\varepsilon}=1-\chi_{\varepsilon}$. Using (8.9), (8.10), (8.11) with $\tilde{f}=\chi_{\varepsilon} \varphi_{\jmath}$, we have

$$
\begin{gathered}
\left|I_{0}(\varepsilon)\right| \leqq C \varepsilon\left\|\varphi_{j}\right\|_{p, \varepsilon} \leqq C \varepsilon \\
\left|I_{3}(\varepsilon)\right| \leqq C\left(|g(\varepsilon)| \varepsilon^{\sigma}+|h(\varepsilon)| \varepsilon^{\sigma}+|i(\varepsilon)| \varepsilon^{\sigma} \varepsilon^{-2 / p}\right)\left\|\varphi_{j}\right\|_{p, \varepsilon} \\
\leqq C\left(\varepsilon+\varepsilon^{2+\sigma}+\varepsilon^{4+\sigma-2 / p}\right) \\
\leqq C\left(\varepsilon+\varepsilon^{2+\sigma}\right) \quad \text { for } p>2 .
\end{gathered}
$$

Since $G \varphi_{j}(x)=\mu_{j}^{-1} \varphi_{j}(x)$, we have

$$
\left|I_{1}(\varepsilon)\right| \leqq C \varepsilon^{2} .
$$

Furthermore, we have the following estimation (8.16) in p. 267 of Ozawa [7].

$$
\left|I_{2}(\varepsilon)\right| \leqq C \varepsilon^{2}|\log \varepsilon| .
$$

Summing up (8.12), (8.13), (8.14), (8.15) and (8.16), we have

$$
\begin{aligned}
\left|v_{\varepsilon}(x)-k \varepsilon^{\sigma} \frac{\partial v_{\varepsilon}}{\partial x_{1}}(x)\right|_{x=(\varepsilon, 0)} \mid & \leqq C\left(\varepsilon+\varepsilon^{\sigma}\left(\varepsilon^{2}+\varepsilon^{2}|\log \varepsilon|\right)+\varepsilon+\varepsilon^{2+\sigma}\right) \\
& \leqq C\left(\varepsilon+\varepsilon^{2+\sigma}|\log \varepsilon|\right) .
\end{aligned}
$$


By Lemma 3.1, we have

$$
\left\|v_{\varepsilon}\right\|_{2, \varepsilon} \leqq C \varepsilon^{1-\sigma}\left(\varepsilon+\varepsilon^{2+\sigma}|\log \varepsilon|\right)=C \cdot H(\varepsilon) .
$$

Here,

$$
\begin{aligned}
H(\varepsilon) & =\varepsilon^{2-\sigma}+\varepsilon^{3}|\log \varepsilon| \\
& \leqq C \varepsilon^{2-\sigma} \quad(-1<\sigma<0) \\
& \leqq C \varepsilon^{3}|\log \varepsilon| \quad(\sigma \leqq-1) .
\end{aligned}
$$

Therefore, we get Theorem 4 .

9. Convergence of eigenvalues for $\sigma<0$.

We introduce the following kernel $\tilde{p}_{\varepsilon}(x, y)$.

$$
\begin{aligned}
\tilde{p}_{\varepsilon}(x, y)=G(x, y) & +g(\varepsilon) G(x, \tilde{w}) G(\tilde{w}, y) \\
& +h(\varepsilon)\left\langle\nabla_{w} G(x, \tilde{w}), \nabla_{w} G(\tilde{w}, y)\right\rangle \chi_{\varepsilon}(x) \chi_{\varepsilon}(y) \\
& +i(\varepsilon)\left\langle H_{w} G(x, \tilde{w}), H_{w} G(\tilde{w}, y)\right\rangle \chi_{\varepsilon}(x) \chi_{\varepsilon}(y)
\end{aligned}
$$

And we put

$$
\left(\tilde{\boldsymbol{P}}_{\varepsilon} f\right)(x)=\int_{\Omega} \tilde{p}_{\varepsilon}(x, y) f(y) d y .
$$

Notice that $\left(1-\chi_{\varepsilon}\right) \chi_{\varepsilon}=0$ in $h(\varepsilon)$-term and $\imath(\varepsilon)$-term of $(9.1)$. Therefore, as we get Lemma 5.1, we get the following.

LEMMA 9.1. There exists a constant $C$ independent of $\varepsilon$ such that

$$
\begin{aligned}
\left\|\boldsymbol{P}_{\varepsilon}-\chi_{\varepsilon} \tilde{\boldsymbol{P}}_{\varepsilon} \chi_{\varepsilon}\right\|_{2} \leqq C(\varepsilon+|g(\varepsilon)| \varepsilon|\log \varepsilon|) \\
\\
\leqq C \varepsilon .
\end{aligned}
$$

holds.

Next we want to estimate $\left\|\tilde{\boldsymbol{P}}_{\varepsilon}-\boldsymbol{G}\right\|_{2}$. We take an arbitrary $v \in L^{p}(\boldsymbol{\Omega})$. Then, we see that

$$
\begin{aligned}
\left(\left(\tilde{\boldsymbol{P}}_{\varepsilon}-\boldsymbol{G}\right) v\right)(x)= & g(\varepsilon) G(x, \tilde{w})(\boldsymbol{G} v)(\tilde{w}) \\
& +h(\varepsilon)\left\langle\nabla_{w} G(x, \tilde{w}), \nabla_{w}\left(\boldsymbol{G} \chi_{\varepsilon} v\right)(\tilde{w})\right\rangle \chi_{\boldsymbol{\varepsilon}}(x) \\
& +i(\varepsilon)\left\langle H_{w} G(x, \tilde{w}), H_{w}\left(\boldsymbol{G} \chi_{\varepsilon} v\right)(\tilde{w})\right\rangle \chi_{\varepsilon}(y) .
\end{aligned}
$$

Therefore, 


$$
\begin{aligned}
& \left\|\left(\tilde{\boldsymbol{P}}_{\varepsilon}-\boldsymbol{G}\right) v\right\|_{p} \\
& \leqq \\
& \quad+|g(\boldsymbol{\varepsilon})|\|G(\cdot, w)\|_{p}\|\boldsymbol{G} v\|_{\infty} \\
& \quad+|h(\varepsilon)| \sum_{n=1}^{2}\left(\int_{\Omega_{\varepsilon}}\left|\frac{\partial}{\partial w_{n}} G(x, \tilde{w})\right|^{p} d x\right)^{1 / p}\left|\frac{\partial}{\partial w_{n}}\left(\boldsymbol{G} \chi_{\varepsilon} v\right)(\tilde{w})\right| \\
& \quad+|i(\varepsilon)| \sum_{m, n=1}^{2}\left(\int_{\Omega_{\varepsilon}}\left|\frac{\partial^{2}}{\partial w_{m} \partial w_{n}} G(x, \tilde{w})\right|^{p} d x\right)^{1 / p}\left|\frac{\partial^{2}}{\partial w_{m} \partial w_{n}}\left(\boldsymbol{G} \chi_{\varepsilon} v\right)(\tilde{w})\right|
\end{aligned}
$$

holds for $p<1$.

We have

for $n=1,2$, and

$$
\begin{aligned}
\|\boldsymbol{G} v\|_{\infty} \leqq C\|v\|_{p} & (p>1) \\
\left(\int_{\Omega_{\varepsilon}}\left|\frac{\partial}{\partial w_{n}} G(x, \tilde{w})\right|^{p} d x\right)^{1 / p} & \leqq C\left(\int_{\Omega_{i}}|x-\tilde{w}|^{-p} d x\right)^{1 / p} \\
& \leqq C|\log \varepsilon|^{1 / 2} \quad(\boldsymbol{p}=2) \\
& \leqq C \varepsilon^{2 / p-1} \quad(p>2)
\end{aligned}
$$

$$
\begin{aligned}
\left(\int_{\Omega_{\varepsilon}}\left|\frac{\partial^{2}}{\partial w_{m} \partial w_{n}} G(x, \tilde{w})\right|^{p} d x\right)^{1 / p} & \leqq C\left(\int_{\Omega_{\varepsilon}}|x-\tilde{w}|^{-2 p} d x\right)^{1 / p} \\
& \leqq C \varepsilon^{2 / p-2} \quad(p>1)
\end{aligned}
$$

for $1 \leqq m, n \leqq 2$.

By (9.3), (9.4), (9.5), (9.6) and using the estimation (8.10), (8.11) with $\tilde{f}=\chi_{\varepsilon} v$, we see that

$$
\begin{aligned}
\left\|\left(\tilde{\boldsymbol{P}}_{\varepsilon}-\boldsymbol{G}\right) v\right\|_{2} \leqq C\left(|g(\varepsilon)|\|v\|_{2}+|h(\varepsilon)||\log \varepsilon|^{1 / 2}|\log \varepsilon|^{1 / 2}\|v\|_{2, \varepsilon}\right. \\
\left.+|i(\varepsilon)| \varepsilon^{-1} \varepsilon^{-1}\|v\|_{2, \varepsilon}\right) . \\
\leqq C\left(\varepsilon^{1-\sigma}+\varepsilon^{2}|\log \varepsilon|+\varepsilon^{2}\right)\|v\|_{2} \\
\leqq C\left(\varepsilon^{1-\sigma}+\varepsilon^{2}|\log \varepsilon|\right)\|v\|_{2}
\end{aligned}
$$

holds for an arbitrary $v \in L^{2}(\Omega)$. Therefore, we get the following.

LEMMA 9.2. There exists a constant $C$ independent of $\varepsilon$ such that

$$
\left\|\boldsymbol{P}_{\varepsilon}-\boldsymbol{G}\right\|_{2} \leqq C\left(\varepsilon^{1-\sigma}+\varepsilon^{2}|\log \varepsilon|\right)
$$

holds.

Notice that the $j$-th eigenvalue of $\boldsymbol{P}_{\varepsilon}$ is equal to the $\}$-th eigenvalue of $\chi_{\varepsilon} \tilde{\boldsymbol{P}}_{\varepsilon} \chi_{\varepsilon}$. We fix $\beta \in(0,1)$. Then, by virtue of Proposition 8.1, Lemma 9.1 and 9.2 , we see that there exists a constant $C$ independent of $j$ such that

$$
\left|\mu_{j}(\varepsilon)^{-1}-\mu_{j}^{-1}\right| \leqq C\left(\varepsilon^{1+\beta}+\varepsilon+\varepsilon^{1-\sigma}+\varepsilon^{2}|\log \varepsilon|\right) \leqq C \varepsilon
$$


holds.

We need more precise estimate estimate for the left hand side of (9.7) to get Theorem 2. By (9.7), we know that the multiplicity of $\mu_{j}(\varepsilon)$ is one for small $\varepsilon$ when the multiplicity of $\mu_{\rho}$ is one.

\section{Perturbational Calculus for $\boldsymbol{P}_{\varepsilon}$.}

In this section we consider the behaviour of eigenvalues of $\boldsymbol{P}_{\boldsymbol{\varepsilon}}$ as $\boldsymbol{\varepsilon}$ tends to 0 . We put $A_{0}, A_{1}$ as before. And we put

$$
\begin{aligned}
& \left(A_{2} f\right)(x)=\left\langle\nabla_{w} G(x, \tilde{w}), \nabla_{w}\left(G \xi_{\varepsilon} f\right)(\tilde{w})\right\rangle \xi_{\varepsilon}(x) \\
& \left(A_{3} f\right)(x)=\left\langle H_{w} G(x, \tilde{w}), H_{w}\left(G \xi_{\varepsilon} f\right)(\tilde{w})\right\rangle \xi_{\varepsilon}(x) .
\end{aligned}
$$

Then,

$$
\overline{\boldsymbol{P}_{\varepsilon}}=A_{0}+\bar{g}(\boldsymbol{\varepsilon}) A_{1}+h(\boldsymbol{\varepsilon}) A_{2}+i(\boldsymbol{\varepsilon}) A_{3} .
$$

where

$$
\bar{g}(\varepsilon)=g(\varepsilon)-\pi \mu_{j} \varepsilon^{2}
$$

Furthermore, we put

$$
\begin{aligned}
& \lambda(\varepsilon)=\lambda_{0}+\bar{g}(\varepsilon) \lambda_{1}+h(\varepsilon) \lambda_{2}+i(\varepsilon) \lambda_{3} \\
& \phi(\varepsilon)=\psi_{0}+\bar{g}(\varepsilon) \psi_{1}+h(\varepsilon) \psi_{2}+i(\varepsilon) \psi_{3}
\end{aligned}
$$

so that $\lambda(\varepsilon)$ and $\phi(\varepsilon)$ is an approximate eigenvalue of $\overline{\boldsymbol{P}}_{\varepsilon}$ and an approximate eigenfunction of $\overline{\boldsymbol{P}_{\varepsilon}}$, respectively.

Let $\lambda_{0}$ be a simple eigenvalue of $A_{0}$. At first we set

$$
\left(A_{0}-\lambda_{0}\right) \phi_{0}=0, \quad\left\|\phi_{0}\right\|_{2}=1
$$

Next we solve the following equations:

$$
\begin{array}{ll}
\left(A_{0}-\lambda_{0}\right) \psi_{1}=\left(\lambda_{1}-A_{1}\right) \psi_{0}, & \left(\psi_{0}, \psi_{1}\right)_{2}=0 \\
\left(A_{0}-\lambda_{0}\right) \psi_{2}=\left(\lambda_{2}-A_{2}\right) \psi_{0}, & \left(\psi_{0}, \phi_{2}\right)_{2}=0 \\
\left(A_{0}-\lambda_{0}\right) \psi_{3}=\left(\lambda_{3}-A_{3}\right) \psi_{0}, & \left(\psi_{0}, \phi_{3}\right)_{2}=0
\end{array}
$$

where $(,)_{2}$ denotes the inner product on $L^{2}(\Omega)$. By the Fredholm alternative theory we see that

$$
\lambda_{n}=\left(A_{n} \psi_{0}, \phi_{0}\right)_{2} \quad(n=1,2,3)
$$

is the condition such that the unique solution $\phi_{1}, \psi_{2}, \phi_{3}$ of (10.3), (10.4), (10.5) exists, respectively.

Hereafter we put $\lambda_{0}=\mu_{j}^{-1}$. Then $\phi_{0}=\varphi_{j}$.

We have the following: 
LEMMA 10.1. For a constant $C$ independent of $\varepsilon$,

hold.

$$
\begin{aligned}
\left\|A_{1}\right\|_{p} & \leqq C \quad(p>1) \\
\left\|A_{2}\right\|_{p} & \leqq C|\log \varepsilon| \quad(p=2) \\
& \leqq C \xi^{2 / p-1} \quad(p>2) \\
\left\|A_{3}\right\|_{p} & \leqq C \varepsilon^{-2} \quad(p>1)
\end{aligned}
$$

Proof. The same estimate as (9.4), (9.5) and (9.6) yields

$$
\begin{aligned}
\left\|A_{1} f\right\|_{p} & \leqq C\|f\|_{p} \quad(p>1) \\
\left\|A_{2} f\right\|_{p} & \leqq \sum_{n=1}^{2}\left(\int_{\Omega \backslash B_{\varepsilon / 2}}\left|\frac{\partial}{\partial w_{n}} G(x, \tilde{w})\right|^{p} d x\right)^{1 / p}\left|\frac{\partial}{\partial w_{n}}\left(\boldsymbol{G} \boldsymbol{\xi}_{\varepsilon} f\right)(\tilde{w})\right| \\
& \leqq C|\log \varepsilon|\|f\|_{2} \quad(p=2) \\
& \leqq C \xi^{2 / p-1}\|f\|_{p} \quad(p>2) \\
\left\|A_{3} f\right\|_{p} & \leqq \sum_{m, n=1}^{2}\left(\int_{\Omega \backslash B_{\varepsilon / 2}}\left|\frac{\partial^{2}}{\partial w_{m} \partial w_{n}} G(x, \tilde{w})\right|^{p} d x\right)^{1 / p}\left|\frac{\partial^{2}}{\partial w_{m} \partial w_{n}}(\boldsymbol{G} \xi f)(\tilde{w})\right| \\
& \leqq C \varepsilon^{-2}\|f\|_{p} \quad(p>1),
\end{aligned}
$$

because $\xi_{\varepsilon}(x)=0$ for $x \in B_{\varepsilon / 2}$. Therefore, we get the desired result. q.e.d..

By (10.6) we see that

$$
\left|\lambda_{n}\right| \leqq\left|\left(A_{n} \psi_{0}, \psi_{0}\right)_{2}\right| \leqq C\left\|A_{n}\right\| p \quad(n=1,2,3)
$$

for $p>1$.

Then, by the Fredholm theory and the estimate of the $L^{p}(\Omega)$ norm of the right hand side of (10.3), (10.4) and (10.5), we get the following

Lemma 10.2. For a constant $C$ independent of $\varepsilon$,

$$
\begin{aligned}
\left\|\phi_{1}\right\|_{p} & \leqq C \quad(p>1) \\
\left\|\psi_{2}\right\|_{p} & \leqq C|\log \varepsilon| \quad(p=2) \\
& \leqq C \varepsilon^{2 / p-1} \quad(p>2) \\
\left\|\psi_{3}\right\|_{p} & \leqq C \varepsilon^{-2} \quad(p>1)
\end{aligned}
$$

hold.

In view of (10.2), (10.3), (10.4) and (10.5), we have 


$$
\begin{aligned}
\left(\overline{\boldsymbol{P}}_{\varepsilon}-\lambda(\varepsilon)\right) \psi(\varepsilon)= & \bar{g}(\varepsilon)^{2}\left(A_{1}-\lambda_{1}\right) \psi_{1}+h(\varepsilon)^{2}\left(A_{2}-\lambda_{2}\right) \psi_{2}+i(\varepsilon)^{2}\left(A_{3}-\lambda_{3}\right) \psi_{3} \\
& +\bar{g}(\varepsilon) h(\varepsilon)\left(\left(A_{1}-\lambda_{1}\right) \psi_{2}+\left(A_{2}-\lambda_{2}\right) \psi_{1}\right) \\
& +h(\varepsilon) i(\varepsilon)\left(\left(A_{2}-\lambda_{2}\right) \psi_{3}+\left(A_{3}-\lambda_{3}\right) \phi_{2}\right) \\
& +i(\varepsilon) \bar{g}(\varepsilon)\left(\left(A_{3}-\lambda_{3}\right) \psi_{1}+\left(A_{1}-\lambda_{1}\right) \psi_{3}\right) .
\end{aligned}
$$

By (10.7), (10.8), Lemmas 10.1 and 10.2 , we see that

$$
\begin{aligned}
\left\|\left(\overline{\boldsymbol{P}}_{\varepsilon}-\lambda(\varepsilon)\right) \psi(\varepsilon)\right\|_{2} & \leqq C\left(\bar{g}(\varepsilon)^{2}+\varepsilon^{4}|\log \varepsilon|^{2}+|\bar{g}(\varepsilon)| \varepsilon^{2}|\log \varepsilon|\right) \\
& \leqq C\left(|\bar{g}(\varepsilon)|+\varepsilon^{2}|\log \varepsilon|\right)^{2} .
\end{aligned}
$$

By (10.1) we have

$$
\begin{aligned}
& \left(|\bar{g}(\varepsilon)|+\varepsilon^{2}|\log \varepsilon|\right)^{2} \\
& \quad \leqq C\left(\varepsilon^{1-\sigma}+\varepsilon^{2}|\log \varepsilon|\right)^{2} \leqq C\left(\varepsilon^{2-\sigma}+\varepsilon^{3}|\log \varepsilon|\right)=C \cdot H(\varepsilon) .
\end{aligned}
$$

Therefore, we get the following.

Proposition 10.3. There exists a constant $C$ independent of $\varepsilon$ such that

$$
\left\|\left(\overline{\boldsymbol{P}_{\varepsilon}}-\lambda(\varepsilon)\right) \phi(\varepsilon)\right\|_{2} \leqq C \cdot H(\varepsilon)
$$

holds.

Furthermore we want to estimate $\left\|\left(\boldsymbol{P}_{\varepsilon}-\boldsymbol{G}_{\varepsilon}\right)\left(\chi_{\varepsilon} \psi(\varepsilon)\right)\right\|_{2, \varepsilon}$. We fix $\beta \in(0,1)$. Then, by Proposition 8.1, Lemma 10.2, Theorem 4 and (10.1), we have

$$
\begin{aligned}
&\left\|\left(\boldsymbol{P}_{\varepsilon}-\boldsymbol{G}_{\varepsilon}\right)\left(\chi_{\varepsilon} \psi(\varepsilon)\right)\right\|_{2} \\
& \leqq\left\|\left(\boldsymbol{P}_{\varepsilon}-\boldsymbol{G}_{\varepsilon}\right)\left(\chi_{\varepsilon} \varphi_{j}\right)\right\|_{2}, \\
&+\left\|\boldsymbol{P}_{\varepsilon}-\boldsymbol{G}_{\varepsilon}\right\|_{2, \varepsilon}\left(|\bar{g}(\varepsilon)|\left\|\phi_{1}\right\|_{2}+|h(\varepsilon)|\left\|\phi_{2}\right\|_{2}+|i(\varepsilon)|\left\|\phi_{3}\right\|_{2}\right) \\
& \leqq C\left(H(\varepsilon)+\varepsilon^{1+\beta}\left(\varepsilon^{1-\sigma}+\varepsilon^{2}|\log \varepsilon|\right)\right) \\
&= C\left(1+\varepsilon^{\beta}\right) H(\varepsilon) \leqq C \cdot H(\varepsilon) .
\end{aligned}
$$

Therefore, we get the following.

Proposition 10.4. There exists a constant $C$ independent of $\varepsilon$ such that

$$
\left\|\left(\boldsymbol{P}_{\varepsilon}-\boldsymbol{G}_{\varepsilon}\right)\left(\chi_{\varepsilon} \psi(\varepsilon)\right)\right\|_{2, \varepsilon} \leqq C \cdot H(\varepsilon)
$$

holds.

\section{Proof of Theorem 5 .}

We put 


$$
J_{\varepsilon}(x ; v)=\left(\chi_{\varepsilon} \overline{\boldsymbol{P}}_{\varepsilon} v-\boldsymbol{P}_{\varepsilon} \chi_{\varepsilon} v\right)(x) \quad \text { for } v \in L^{p}(\boldsymbol{\Omega}) .
$$

Then, we see that

$$
\begin{aligned}
& \Delta J_{\varepsilon}(x ; v)=0 \quad x \in \Omega_{\varepsilon} \\
& J_{\varepsilon}(x ; v)=0 \quad x \in \partial \Omega .
\end{aligned}
$$

As we get (8.8), we have

$$
\begin{aligned}
& J_{\varepsilon}(x ; v)-\left.k \varepsilon^{\sigma} \frac{\partial}{\partial x_{1}} J_{\varepsilon}(x ; v)\right|_{x=(\varepsilon, 0)} \\
& \quad=\sum_{n=4}^{6} I_{n}(\varepsilon ; v)+\sum_{n=8}^{9} I_{n}(\varepsilon ; v)-k \varepsilon^{\sigma}\left(I_{7}(\varepsilon ; v)+I_{10}(\varepsilon ; v)\right)
\end{aligned}
$$

where

$$
\begin{aligned}
I_{4}(\varepsilon ; v)= & \left(\boldsymbol{G} \hat{\chi}_{\varepsilon} v\right)(x)-\left(\boldsymbol{G} \hat{\chi}_{\varepsilon} v\right)(\tilde{w})-\varepsilon \frac{\partial}{\partial w_{1}}\left(\boldsymbol{G} \xi_{\varepsilon} \hat{\chi}_{\varepsilon} v\right)(\tilde{w}) \\
I_{5}(\varepsilon ; v)= & g(\varepsilon)\left(O(\varepsilon)-k \boldsymbol{\varepsilon}^{\sigma} \frac{\partial}{\partial x_{1}} S(x, \tilde{w})\right)\left(\boldsymbol{G} \hat{\chi}_{\varepsilon} v\right)(\tilde{w}) \\
I_{6}(\varepsilon ; v)= & -\pi \mu_{j} \varepsilon^{2} G(x, \tilde{w})(\boldsymbol{G} v)(\tilde{w}) \\
I_{7}(\varepsilon ; v)= & \frac{\partial}{\partial x_{1}}\left(\boldsymbol{G} \hat{\chi}_{\varepsilon} v\right)(x)-\left(\frac{\partial}{\partial w_{1}}+\varepsilon \frac{\partial^{2}}{\partial w_{1}^{2}}\right)\left(\boldsymbol{G} \xi_{\varepsilon} \hat{\chi}_{\varepsilon} v\right)(\tilde{w}) \\
I_{8}(\varepsilon ; v)= & h(\varepsilon)\left\langle\nabla_{w} S(x, \tilde{w}), \nabla_{w}\left(\boldsymbol{G} \xi_{\varepsilon} \hat{\chi}_{\varepsilon} v\right)(\tilde{w})\right\rangle \\
& -k \varepsilon^{\sigma} h(\varepsilon) \frac{\partial}{\partial x_{1}}\left\langle\nabla_{w} S(x, w), \nabla_{w}\left(\boldsymbol{G} \xi_{\varepsilon} \hat{\chi}_{\varepsilon} v\right)(\tilde{w})\right\rangle \\
I_{9}(\varepsilon ; v)= & i(\varepsilon)\left\langle H_{w} S(x, \tilde{w}), H_{w}\left(\boldsymbol{G} \xi_{\varepsilon} \hat{\chi}_{\varepsilon} v\right)(\tilde{w})\right\rangle \\
& -k \varepsilon^{\sigma} i(\varepsilon) \frac{\partial}{\partial x_{1}}\left\langle H_{w} S(x, \tilde{w}), H_{w}\left(\boldsymbol{G} \xi_{\varepsilon} \hat{\chi}_{\varepsilon} v\right)(\tilde{w})\right\rangle \\
I_{10}(\varepsilon ; v)= & -\pi \mu_{j} \varepsilon^{2} \frac{\partial}{\partial x_{1}} G(x, \tilde{w})(\boldsymbol{G} v)(\tilde{w})
\end{aligned}
$$

for $x=(\varepsilon ; 0), \tilde{w}=(0,0)$.

By the Sobolev embedding theorem, we have

$$
\begin{aligned}
\left|I_{4}(\varepsilon ; v)\right| & \leqq C \varepsilon\left\|\hat{\chi}_{\varepsilon} v\right\|_{p}+C \varepsilon\left\|\xi_{\varepsilon} \hat{\chi}_{\varepsilon} v\right\|_{p} \\
& \leqq C \varepsilon\|v\|_{p} \quad(p>2) .
\end{aligned}
$$

Also,

$$
\begin{aligned}
\left|I_{5}(\varepsilon ; v)\right| & \leqq C|g(\varepsilon)| \varepsilon^{\sigma}\left(\int_{B_{\varepsilon}}|\log | y-w||^{p^{\prime}} d y\right)^{1 / p^{\prime}}\|v\|_{p} \\
& \leqq C \varepsilon^{3-2 / p}|\log \varepsilon|\|v\|_{p} \quad(p>1)
\end{aligned}
$$




$$
\begin{aligned}
\left|I_{6}(\varepsilon ; v)\right| & \leqq C \varepsilon^{2}|\log \varepsilon|\|v\|_{p} \quad(p>1) \\
\left|I_{10}(\varepsilon ; v)\right| & \leqq C \varepsilon\|v\|_{p} \quad(p>1) \\
\left|I_{8}(\varepsilon ; v)\right| & \leqq C|h(\varepsilon)| \varepsilon^{\sigma}\left(\int_{B_{\varepsilon} \backslash B_{\varepsilon / 2}}|y-w|^{-p^{\prime}} d y\right)^{1 / p^{\prime}}\|v\|_{p} \\
& \leqq C \varepsilon^{3+\sigma-2 / p}\|v\|_{p} \quad(p>2) \\
\left|I_{9}(\varepsilon ; v)\right| & \leqq C|i(\varepsilon)| \varepsilon^{\sigma}\left(\int_{B_{\varepsilon} \backslash B_{\varepsilon / 2}}|y-w|^{-2 p^{\prime}} d y\right)^{1 / p^{\prime}}\|v\|_{p} \\
& \leqq C \varepsilon^{4+\sigma-2 / p}\|v\|_{p} \quad(p>1),
\end{aligned}
$$

where $p^{\prime}$ satisfies $(1 / p)+\left(1 / p^{\prime}\right)=1$.

Since $B(\varepsilon, w) \subset B(2 \varepsilon, x)$ for $x=(\varepsilon, 0)$ and $\tilde{w}=(0,0)$,

$$
\begin{aligned}
\left|I_{7}(\varepsilon ; v)\right| \leqq & C\left(\int_{B(2 \varepsilon, x)}|x-y|^{-p^{\prime}} d y\right)^{1 / p^{\prime}}\|v\|_{p} \\
& +C\left(\int_{B_{\varepsilon} \backslash B_{\varepsilon / 2}}|y-w|^{-p^{\prime}} d y\right)^{1 / p^{\prime}}\|v\|_{p} \\
& +C \varepsilon\left(\int_{B_{\varepsilon} \backslash B_{\varepsilon / 2}}|y-w|^{-2 p^{\prime}} d y\right)^{1 / p^{\prime}}\|v\|_{p} \\
\leqq & C \varepsilon^{1-2 / p}\|v\|_{p} \quad(p>2) .
\end{aligned}
$$

Summing up these facts, we have

$$
\begin{gathered}
\left|J_{\varepsilon}(x ; v)-k \varepsilon^{\sigma} \frac{\partial}{\partial x_{1}} J_{\varepsilon}(x ; v)\right|_{x=(\varepsilon, 0)} \mid \\
\leqq C \varepsilon^{1+\sigma-2 / p}\|v\|_{p} \quad(p>2) .
\end{gathered}
$$

By (11.2), (11.11) and Lemma 3.1, we have

$$
\left\|J_{\varepsilon}(\cdot ; v)\right\|_{2, \varepsilon} \leqq C \varepsilon^{2-2 \uparrow}\|\| v \|_{p} \quad(p>2) .
$$

Therefore we get the following.

LEMmA 11.1. There exists a constant $C$ independent of $\varepsilon$ such that

$$
\left\|J_{\varepsilon}(\cdot ; v)\right\|_{2, \varepsilon} \leqq C \varepsilon^{2-2 / p}\|v\|_{p}
$$

holds for any $v \in L^{p}(\Omega)(p>2)$.

By the way, we have the following formula (11.13) in p. 271 of Ozawa [7].

$$
I_{7}\left(\varepsilon ; \varphi_{j}\right)=-(\varepsilon / 2) \varphi_{j}(w)+O\left(\varepsilon^{2}|\log \varepsilon|\right)
$$

It is easy to see 


$$
I_{10}\left(\varepsilon ; \varphi_{j}\right)=(\varepsilon / 2) \varphi_{j}(\tilde{w})+O\left(\varepsilon^{2}\right) \text {. }
$$

Thus, we have

$$
\left|I_{7}\left(\varepsilon ; \varphi_{j}\right)+I_{10}\left(\varepsilon ; \varphi_{j}\right)\right| \leqq C \varepsilon^{2}|\log \varepsilon| .
$$

Summing up (11.3), (11.4), (11.5), (11.6), (11.8), (11.9) and (11.15), we have

$$
\begin{aligned}
& \left|J_{\varepsilon}\left(x ; \varphi_{j}\right)-k \varepsilon^{\sigma} \frac{\partial}{\partial x_{1}} J_{\varepsilon}\left(x ; \varphi_{j}\right)\right|_{x=(\varepsilon, 0)} \mid \\
& \quad \leqq C\left(\varepsilon+\varepsilon^{2+\sigma}|\log \varepsilon|\right) .
\end{aligned}
$$

By (11.16) and Lemma 3.1, we have

$$
\left\|J_{\varepsilon}\left(\cdot ; \varphi_{j}\right)\right\|_{2, \varepsilon} \leqq C \varepsilon^{1-\sigma}\left(\varepsilon+\varepsilon^{2+\sigma}|\log \varepsilon|\right)=C \cdot H(\varepsilon) .
$$

Therefore we get Theorem 5 .

Furthermore we want to estimate $\left\|J_{\varepsilon}(\cdot ; \phi(\varepsilon))\right\|_{2, \varepsilon}$. By (11.17), Lemmas 10.2 and 11.1 , we have

$$
\begin{aligned}
\left\|J_{\varepsilon}(\cdot ; \phi(\varepsilon))\right\|_{2, \varepsilon} \leqq & \left\|J_{\varepsilon}\left(\cdot ; \varphi_{j}\right)\right\|_{2, \varepsilon}+|\bar{g}(\varepsilon)|\left\|J_{\varepsilon}\left(\cdot ; \phi_{1}\right)\right\|_{2, \varepsilon} \\
& +\left|h(\varepsilon)\left\|J_{\varepsilon}\left(\cdot ; \phi_{2}\right)\right\|_{2, \varepsilon}+\right| i(\varepsilon) \mid\left\|J_{\varepsilon}\left(\cdot ; \phi_{3}\right)\right\|_{2, \varepsilon} \\
\leqq & C\left(\varepsilon^{2-\sigma}+\varepsilon^{3}|\log \varepsilon|+\varepsilon^{3-\sigma-2 / p}+\varepsilon^{3}+\varepsilon^{4-2 / p}\right) \\
\leqq & C\left(\varepsilon^{2-\sigma}+\varepsilon^{3}|\log \varepsilon|\right)=C \cdot H(\varepsilon) \quad \text { for } p>2 .
\end{aligned}
$$

Therefore we get the following.

Proposition 11.2. There exists a constant $C$ independent of $\varepsilon$ such that

$$
\left\|\left(\boldsymbol{P}_{\varepsilon} \chi_{\varepsilon}-\chi_{\varepsilon} \overline{\boldsymbol{P}}_{\varepsilon}\right) \psi(\varepsilon)\right\|_{2, \varepsilon} \leqq C \cdot H(\varepsilon)
$$

holds.

\section{Proof of Theorem 2.}

Now we are in a position to prove Theorem 2. By Propositions 10.3, 10.4 and 11.2 , we have

$$
\left\|\left(\boldsymbol{G}_{\varepsilon}-\lambda(\varepsilon)\right)\left(\chi_{\varepsilon} \psi(\varepsilon)\right)\right\|_{2, \varepsilon} \leqq C \cdot H(\varepsilon) .
$$

Notice that $\|\psi(\varepsilon)\|_{2, \varepsilon} \in(1 / 2,2)$ for small $\varepsilon$.

Therefore, there exists at least one eigenvalue $\lambda^{*}(\varepsilon)$ of $\boldsymbol{G}_{\varepsilon}$ satisfying

$$
\left|\lambda^{*}(\varepsilon)-\lambda(\varepsilon)\right| \leqq C \cdot H(\varepsilon) \text {. }
$$

We here represent $\lambda_{1}, \lambda_{2}, \lambda_{3}$ as follows: 


$$
\begin{aligned}
\lambda_{1} & =\mid\left(\left.\boldsymbol{G} \phi_{0}(\tilde{w})\right|^{2}=\mu_{j}^{-2} \varphi_{j}(\tilde{w})^{2}\right. \\
\lambda_{2} & =\left\langle\nabla_{w}\left(\boldsymbol{G} \xi_{\varepsilon} \psi_{0}\right)(\tilde{w}), \nabla_{w}\left(\boldsymbol{G} \xi_{\varepsilon} \psi_{0}\right)(\tilde{w})\right\rangle \\
& =\left.\sum_{n=1}^{2}\left(\frac{\partial}{\partial w_{n}} \int_{\Omega} G(w, y) \xi_{\varepsilon}(y) \varphi_{j}(y) d y\right)^{2}\right|_{w=\tilde{w}} \\
\lambda_{3} & =\left\langle H_{w}\left(\boldsymbol{G} \xi_{\varepsilon} \psi_{0}\right)(\tilde{w}), H_{w}\left(\boldsymbol{G} \xi_{\varepsilon} \psi_{0}\right)(\tilde{w})\right\rangle \\
& =\left.\sum_{m, n=1}^{2}\left(\frac{\partial^{2}}{\partial w_{m} \partial w_{n}} \int_{\Omega} G(w, y) \xi_{\varepsilon}(y) \varphi_{j}(y) d y\right)^{2}\right|_{w=\tilde{w}}
\end{aligned}
$$

We see that

$$
\begin{aligned}
& \left|\frac{\partial^{2}}{\partial w_{m} \partial w_{n}} \int_{\Omega} G(w, y) \xi_{\varepsilon}(y) \varphi_{j}(y) d y\right|_{w=\tilde{w}} \mid \\
& \quad \leqq C \int_{\Omega \backslash B_{\varepsilon / 2}}|y-\tilde{w}|^{-2} d y \leqq C|\log \varepsilon| \quad(1 \leqq m, n \leqq 2) .
\end{aligned}
$$

Thus, we have

$$
\lambda_{3}=O\left(|\log \varepsilon|^{2}\right)
$$

Also,

$$
\begin{aligned}
& \left.\frac{\partial}{\partial w_{n}} \int_{\Omega} G(w, y) \xi_{\varepsilon}(y) \varphi_{j}(y) d y\right|_{w=\tilde{w}} \\
& \quad=\mu_{j}^{-1} \frac{\partial}{\partial w_{n}} \varphi_{j}(\tilde{w})+I_{11}^{(n)}(\varepsilon)+I_{12}^{(n)}(\varepsilon),
\end{aligned}
$$

where

$$
\begin{array}{r}
I_{11}^{(n)}(\varepsilon)=-\left.\frac{\partial}{\partial w_{n}} \int_{\Omega} S(w, y)\left(1-\xi_{\varepsilon}(y)\right) \varphi_{j}(y) d y\right|_{w=\tilde{w}} \\
I_{12}^{(n)}(\varepsilon)=-\left.\frac{\partial}{\partial w_{n}} \int_{\Omega} L(w, y)\left(1-\xi_{\varepsilon}(y)\right) \varphi_{j}(y) d y\right|_{w=\tilde{w}} \\
\text { for } n=1,2 .
\end{array}
$$

Here, we put

$$
L(w, y)=G(w, y)-S(w, y)=-(2 \pi)^{-1} \log |w-y| .
$$

We see that

$$
\left|I_{11}^{(n)}(\varepsilon)\right| \leqq C \int_{B_{\varepsilon}} 1 d y \leqq C^{\prime} \varepsilon^{2} \quad(n=1,2) .
$$

Furthermore, we have the following formula (12.8) in p. 271 of Ozawa [7].

$$
\left|I_{12}^{(n)}(\varepsilon)\right| \leqq C \varepsilon^{2}|\log \varepsilon| \quad(n=1,2) .
$$

Summing up (12.3), (12.6), (12.7) and (12.8), we have

$$
\lambda_{2}=\mu_{j}^{-2}\left|\operatorname{grad} \varphi_{j}(\tilde{w})\right|^{2}+O\left(\varepsilon^{2}|\log \varepsilon|\right) .
$$


By (12.2), (12.5) and (12.9), we see that

$$
\begin{aligned}
\lambda(\varepsilon) & =\mu_{\jmath}^{-1}+\bar{g}(\varepsilon) \lambda_{1}+h(\varepsilon) \lambda_{2}+i(\varepsilon) \lambda_{3} \\
& =\mu_{\jmath}{ }^{-1}-\mu_{\jmath}^{-2} Q_{j} \varepsilon^{1-\sigma}-\mu_{\jmath}^{-2} R_{j} \varepsilon^{2}+O\left(\varepsilon^{4}|\log \varepsilon|^{2}\right)+O\left(\varepsilon^{2-2 \sigma}|\log \varepsilon|\right),
\end{aligned}
$$

where $Q$, and $R$, are as mentioned before.

By (12.1), (12.10) and the fact (9.7), we see that $\lambda^{*}(\varepsilon)$ must be $\mu_{j}(\varepsilon)^{-1}$. Then. we have

$$
\begin{aligned}
& \left|\mu_{j}(\varepsilon)^{-1}-\left(\mu_{j}^{-1}-\mu_{j}^{-2} Q_{j} \varepsilon^{1-\sigma}-\mu_{j}^{-2} R_{j} \varepsilon^{2}\right)\right| \\
& \quad \leqq C \cdot H(\varepsilon)+C\left(\varepsilon^{4}|\log \varepsilon|^{2}+\varepsilon^{2-2 \sigma}|\log \varepsilon|\right) \\
& \quad=C\left(\varepsilon^{2-\sigma}+\varepsilon^{3}|\log \varepsilon|+\varepsilon^{4}|\log \varepsilon|^{2}+\varepsilon^{2-2 \sigma}|\log \varepsilon|\right) \\
& \quad \leqq C\left(\varepsilon^{2-\sigma}+\varepsilon^{3}|\log \varepsilon|\right) .
\end{aligned}
$$

Therefore, we get the desired Theorem 2 .

\section{REFERENCFS}

-1] C. AnNé, Spectre du laplacien et écrasement d'ansens, Ann. Sc1. Ecole Norm. Sup., 20 (1987), 271-280.

[2] J.M. Arrieta, J. Hale ANd Q. HaN, Eigenvalue problems for nonsmoothly perturbed domains, J. Diff. Equations., 91 (1991), 24-52.

[3] G. Besson, Comportement asymptotique des valeurs propres du laplacien dans un domaine avec un trou, Bull. Soc. Math. France., 113 (1985), 211-239.

[4] I. Chavel, Eigenvalues in Riemannian geometry, Academic Press (1984).

[5] S. Jімво, The singularly perturbed domain and the characterization for the eigenfunctions with Neumann boundary condition, J. of Diff. Equations., 77 (1989), $322-350$.

[6] S. OzAwA, Singular variation of domain and spectra of the Laplacian with small Robin conditional boundary I to appear in Osaka J. Math. 1992.

[7] S. OzAwA, Spectra of domains with small spherical Neumann boundary, J. Fac. Sci. Univ. Tokyo SecIA., 30 (1983), 259-277.

[8] S. Ozawa, Asymptotic property of an eigenfunction of the Laplacian under singular variation of domains - the Neumann condition-, Osaka J. Math., 22 (1985), 639-655.

[9] S. Ozawa, Electrostatic capacity and eigenvalues of the Laplacian, J. Fac. Sci. Univ. Tokyo SecIA., 30 (1983), 53-62.

[10] J. RAUCH AND M. TAYLOR, Potential and scattering theory on wildly perturbed domains, J. Funct. Anal., 18 (1975), 27-59.

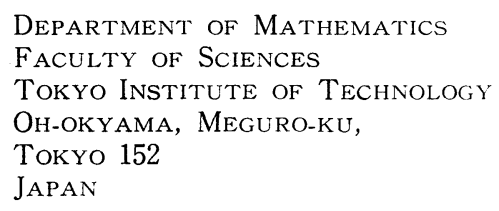

